\title{
Hybrid Steepest-Descent Methods for Triple Hierarchical Variational Inequalities
}

\author{
L. C. Ceng, ${ }^{1}$ A. Latif, ${ }^{2}$ C. F. Wen, ${ }^{3}$ and A. E. Al-Mazrooei ${ }^{4}$ \\ ${ }^{1}$ Department of Mathematics, Shanghai Normal University, and Scientific Computing Key Laboratory of Shanghai Universities, \\ Shanghai 200234, China \\ ${ }^{2}$ Department of Mathematics, King Abdulaziz University, P.O. Box 80203, Jeddah 21589, Saudi Arabia \\ ${ }^{3}$ Center for Fundamental Science, Kaohsiung Medical University, Kaohsiung 807, Taiwan \\ ${ }^{4}$ Department of Mathematics, College of Science, University of Jeddah, P.O. Box 80203, Jeddah 21589, Saudi Arabia
}

Correspondence should be addressed to A. Latif; alatif@kau.edu.sa

Received 28 October 2014; Accepted 5 January 2015

Academic Editor: Mohamed-Aziz Taoudi

Copyright (C) 2015 L. C. Ceng et al. This is an open access article distributed under the Creative Commons Attribution License, which permits unrestricted use, distribution, and reproduction in any medium, provided the original work is properly cited.

We introduce and analyze a relaxed iterative algorithm by combining Korpelevich's extragradient method, hybrid steepest-descent method, and Mann's iteration method. We prove that, under appropriate assumptions, the proposed algorithm converges strongly to a common element of the fixed point set of infinitely many nonexpansive mappings, the solution set of finitely many generalized mixed equilibrium problems (GMEPs), the solution set of finitely many variational inclusions, and the solution set of general system of variational inequalities (GSVI), which is just a unique solution of a triple hierarchical variational inequality (THVI) in a real Hilbert space. In addition, we also consider the application of the proposed algorithm for solving a hierarchical variational inequality problem with constraints of finitely many GMEPs, finitely many variational inclusions, and the GSVI. The results obtained in this paper improve and extend the corresponding results announced by many others.

\section{Introduction}

Let $C$ be a nonempty closed convex subset of a real Hilbert space $H$ and let $P_{C}$ be the metric projection of $H$ onto $C$. Let $S: C \rightarrow H$ be a nonlinear mapping on $C$. We denote by $\operatorname{Fix}(S)$ the set of fixed points of $S$ and by $\mathbf{R}$ the set of all real numbers. A mapping $S: C \rightarrow H$ is called $L$-Lipschitz continuous if there exists a constant $L>0$ such that

$$
\|S x-S y\| \leq L\|x-y\|, \quad \forall x, y \in C .
$$

In particular, if $L=1$, then $S$ is called a nonexpansive mapping; if $L \in(0,1)$, then $S$ is called a contraction.

Let $A: C \rightarrow H$ be a nonlinear mapping on $C$. We consider the following variational inequality problem (VIP): find a point $x \in C$ such that

$$
\langle A x, y-x\rangle \geq 0, \quad \forall y \in C .
$$

The solution set of VIP (2) is denoted by $\operatorname{VI}(C, A)$.
The VIP (2) was first discussed by Lions [1]. In 1976, Korpelevich [2] proposed an iterative algorithm for solving the VIP (2) in Euclidean space $\mathbf{R}^{n}$ :

$$
\begin{gathered}
y_{n}=P_{C}\left(x_{n}-\tau A x_{n}\right), \\
x_{n+1}=P_{C}\left(x_{n}-\tau A y_{n}\right), \quad \forall n \geq 0,
\end{gathered}
$$

with $\tau>0$ a given number, which is known as the extragradient method.

Let $\varphi: C \rightarrow \mathbf{R}$ be a real-valued function, let $A$ : $H \rightarrow H$ be a nonlinear mapping, and let $\Theta: C \times C \rightarrow \mathbf{R}$ be a bifunction. In 2008, Peng and Yao [3] introduced the following generalized mixed equilibrium problem (GMEP) of finding $x \in C$ such that

$$
\Theta(x, y)+\varphi(y)-\varphi(x)+\langle A x, y-x\rangle \geq 0, \quad \forall y \in C .
$$

We denote the set of solutions of GMEP (4) by $\operatorname{GMEP}(\Theta, \varphi, A)$. 
It was assumed in [3] that $\Theta: C \times C \rightarrow \mathbf{R}$ is a bifunction satisfying conditions (A1)-(A4) and $\varphi: C \rightarrow \mathbf{R}$ is a lower semicontinuous and convex function with restriction (B1) or (B2), where

(A1) $\Theta(x, x)=0$ for all $x \in C$;

(A2) $\Theta$ is monotone, that is, $\Theta(x, y)+\Theta(y, x) \leq 0$ for any $x, y \in C$;

(A3) $\Theta$ is upper-hemicontinuous; that is, for each $x, y, z \in$ C,

$$
\limsup _{t \rightarrow 0^{+}} \Theta(t z+(1-t) x, y) \leq \Theta(x, y)
$$

(A4) $\Theta(x, \cdot)$ is convex and lower semicontinuous for each $x \in C$;

(B1) for each $x \in H$ and $r>0$, there exists a bounded subset $D_{x} \subset C$ and $y_{x} \in C$ such that, for any $z \in$ $C \backslash D_{x}$,

$$
\Theta\left(z, y_{x}\right)+\varphi\left(y_{x}\right)-\varphi(z)+\frac{1}{r}\left\langle y_{x}-z, z-x\right\rangle<0 ;
$$

(B2) $C$ is a bounded set.

For a given positive number $r$, let $T_{r}^{(\Theta, \varphi)}: H \rightarrow C$ be the solution set of the auxiliary mixed equilibrium problem; that is, for each $x \in H$,

$$
\begin{aligned}
T_{r}^{(\Theta, \varphi)}(x):= & \{y \in C: \Theta(y, z)+\varphi(z)-\varphi(y) \\
& \left.+\frac{1}{r}\langle y-x, z-y\rangle \geq 0, \forall z \in C\right\} .
\end{aligned}
$$

On the other hand, let $B$ be a single-valued mapping of $C$ into $H$ and let $R$ be a multivalued mapping with $D(R)=C$. Consider the following variational inclusion: find a point $x \in$ $C$ such that

$$
0 \in B x+R x
$$

We denote by $I(B, R)$ the solution set of the variational inclusion (8). It is known that problem (8) provides a convenient framework for the unified study of optimal solutions in many optimization related areas including mathematical programming, complementarity problems, variational inequalities, optimal control, mathematical economics, equilibria and game theory. Let $R: D(R) \subset H \rightarrow 2^{H}$ be a maximal monotone set-valued mapping. We define the resolvent operator $J_{R, \lambda}: H \rightarrow \overline{D(R)}$ associated with $R$ and $\lambda$ as follows:

$$
J_{R, \lambda}=(I+\lambda R)^{-1}, \quad \forall x \in H,
$$

where $\lambda$ is a positive number.

Let $F_{1}, F_{2}: C \rightarrow H$ be two mappings. Consider the following general system of variational inequalities (GSVI) of finding $\left(x^{*}, y^{*}\right) \in C \times C$ such that

$$
\begin{array}{ll}
\left\langle\nu_{1} F_{1} y^{*}+x^{*}-y^{*}, x-x^{*}\right\rangle \geq 0, & \forall x \in C, \\
\left\langle\nu_{2} F_{2} x^{*}+y^{*}-x^{*}, x-y^{*}\right\rangle \geq 0, & \forall x \in C,
\end{array}
$$

where $\nu_{1}>0$ and $\nu_{2}>0$ are two constants. It was considered and studied in [4-6]. In particular, if $F_{1}=F_{2}=A$, then the GSVI (10) reduces to the following problem of finding $\left(x^{*}, y^{*}\right) \in C \times C$ such that

$$
\begin{array}{ll}
\left\langle\nu_{1} A y^{*}+x^{*}-y^{*}, x-x^{*}\right\rangle \geq 0, & \forall x \in C, \\
\left\langle\nu_{2} A x^{*}+y^{*}-x^{*}, x-y^{*}\right\rangle \geq 0, & \forall x \in C,
\end{array}
$$

which is defined by Verma [7] and it is called a new system of variational inequalities (NSVI). Further, if $x^{*}=y^{*}$, additionally, then the NSVI reduces to the classical VIP (2). In 2008, Ceng et al. [6] transformed the GSVI (10) into a fixed point problem in the following way.

Proposition CWY (see [6]). For given $\bar{x}, \bar{y} \in C,(\bar{x}, \bar{y})$ is a solution of the GSVI (10) if and only if $\bar{x}$ is a fixed point of the mapping $G: C \rightarrow C$ defined by

$$
G x=P_{C}\left(I-v_{1} F_{1}\right) P_{C}\left(I-v_{2} F_{2}\right) x, \quad \forall x \in C,
$$

where $\bar{y}=P_{C}\left(I-v_{2} F_{2}\right) \bar{x}$.

In particular, if the mapping $F_{j}: C \rightarrow H$ is $\zeta_{j}$-inverse strongly monotone for $j=1,2$, then the mapping $G$ is nonexpansive provided $v_{j} \in\left(0,2 \zeta_{j}\right]$ for $j=1,2$. We denote by $\operatorname{GSVI}(G)$ the fixed point set of the mapping $G$.

Let $S$ and $T$ be two nonexpansive mappings. In 2009, Yao et al. [8] considered the following hierarchical VIP: find hierarchically a fixed point of $T$, which is a solution to the VIP for monotone mapping $I-S$; namely, find $\tilde{x} \in \operatorname{Fix}(T)$, such that

$$
\langle(I-S) \tilde{x}, p-\tilde{x}\rangle \geq 0, \quad \forall p \in \operatorname{Fix}(T) .
$$

The solution set of the hierarchical VIP (13) is denoted by $\Lambda$. It is not hard to check that solving the hierarchical VIP (13) is equivalent to the fixed point problem of the composite mapping $P_{\mathrm{Fix}(T)} S$; that is, find $\tilde{x} \in C$, such that $\tilde{x}=P_{\operatorname{Fix}(T)} S \tilde{x}$. The authors [8] introduced and analyzed the following iterative algorithm for solving the hierarchical VIP (13):

$$
\begin{gathered}
y_{n}=\beta_{n} S x_{n}+\left(1-\beta_{n}\right) x_{n}, \\
x_{n+1}=\alpha_{n} V x_{n}+\left(1-\alpha_{n}\right) T y_{n}, \quad \forall n \geq 0 .
\end{gathered}
$$

Theorem YLM (see [8, Theorem 3.2]). Let C be a nonempty closed convex subset of a real Hilbert space $H$. Let $S$ and $T$ be two nonexpansive mappings of $C$ into itself. Let $V: C \rightarrow C$ be a fixed contraction with $\alpha \in(0,1)$. Let $\left\{\alpha_{n}\right\}$ and $\left\{\beta_{n}\right\}$ be two sequences in $(0,1)$. For any given $x_{0} \in C$, let $\left\{x_{n}\right\}$ be the sequence generated by (14). Assume that the sequence $\left\{x_{n}\right\}$ is bounded and that

(i) $\sum_{n=0}^{\infty} \alpha_{n}=\infty$;

(ii) $\lim _{n \rightarrow \infty}\left(1 / \alpha_{n}\right)\left|1 / \beta_{n}-1 / \beta_{n-1}\right|=0, \lim _{n \rightarrow \infty}\left(1 / \beta_{n}\right) \mid 1-$ $\alpha_{n-1} / \alpha_{n} \mid=0$

(iii) $\lim _{n \rightarrow \infty} \beta_{n}=0, \lim _{n \rightarrow \infty}\left(\alpha_{n} / \beta_{n}\right)=0$ and $\lim _{n \rightarrow \infty}\left(\beta_{n}^{2} / \alpha_{n}\right)=0$ 
(iv) $\operatorname{Fix}(T) \cap \operatorname{int} C \neq \emptyset$;

(v) there exists a constant $k>0$ such that $\| x-$ $T x \| \geq k \operatorname{Dist}(x, \operatorname{Fix}(T))$ for each $x \in C$, where $\operatorname{Dist}(x, \operatorname{Fix}(T))=\inf _{y \in \operatorname{Fix}(T)}\|x-y\|$. Then, $\left\{x_{n}\right\}$ converges strongly to $\tilde{x}=P_{\Lambda} V \tilde{x}$ which solves the hierarchical VIP

$$
\langle(I-S) \tilde{x}, p-\tilde{x}\rangle \leq 0, \quad \forall p \in \operatorname{Fix}(T) .
$$

Very recently, Iiduka $[9,10]$ considered a variational inequality with a variational inequality constraint over the set of fixed points of a nonexpansive mapping. Since this problem has a triple structure in contrast with bilevel programming problems or hierarchical constrained optimization problems or hierarchical fixed point problem, it is refereed as triple hierarchical constrained optimization problem (THCOP). He presented some examples of THCOP and developed iterative algorithms to find the solution of such a problem. The convergence analysis of the proposed algorithms was also studied in $[9,10]$. Since the original problem is a variational inequality, in this paper, we call it a triple hierarchical variational inequality (THVI). Subsequently, Zeng et al. [11] introduced and considered the following triple hierarchical variational inequality (THVI).

Problem I. Assume that

(i) each $T_{i}: H \rightarrow H$ is a nonexpansive mapping with $\bigcap_{i=1}^{N} \operatorname{Fix}\left(T_{i}\right) \neq \emptyset ;$

(ii) $A_{1}: H \rightarrow H$ is $\alpha$-inverse strongly monotone;

(iii) $A_{2}: H \rightarrow H$ is $\beta$-strongly monotone and $L$ Lipschitz continuous;

(iv) $\operatorname{IV}\left(\bigcap_{i=1}^{N} \operatorname{Fix}\left(T_{i}\right), A_{1}\right) \neq \emptyset$.

Then, the objective is to

$$
\text { find } \begin{aligned}
x^{*} \in \mathrm{VI}\left(\mathrm{VI}\left(\bigcap_{i=1}^{N} \operatorname{Fix}\left(T_{i}\right), A_{1}\right), A_{2}\right) \\
:=\left\{x^{*} \in \mathrm{VI}\left(\bigcap_{i=1}^{N} \operatorname{Fix}\left(T_{i}\right), A_{1}\right):\right. \\
\left\langle A_{2} x^{*}, v-x^{*}\right\rangle \geq 0, \\
\left.\forall v \in \mathrm{VI}\left(\bigcap_{i=1}^{N} \operatorname{Fix}\left(T_{i}\right), A_{1}\right)\right\} .
\end{aligned}
$$

In [11], the authors proposed the following algorithm for solving Problem I.

Algorithm ZWY ([11, Algorithm 3.2]). Let $T_{i}: H \rightarrow H(i=$ $1,2, \ldots, N)$ and $A_{i}: H \rightarrow H(i=1,2)$ satisfy assumptions (i)-(iv) in Problem I. The following steps are presented for solving Problem I.

Step 0. Take $\left\{\alpha_{n}\right\}_{n=0}^{\infty} \subset(0,1],\left\{\rho_{n}\right\}_{n=0}^{\infty} \subset(0,2 \alpha]$, and $\mu \epsilon$ $\left(0,2 \beta / L^{2}\right)$, choose $x_{0} \in H$ arbitrarily, and let $n:=0$.

Step 1. Given $x_{n} \in H$, compute $x_{n+1} \in H$ as

$$
\begin{gathered}
y_{n}=T_{[n+1]}\left(x_{n}-\rho_{n} A_{1} x_{n}\right), \\
x_{n+1}=y_{n}-\mu \alpha_{n} A_{2} y_{n},
\end{gathered}
$$

where $T_{[k]}:=T_{k \bmod N}$, for integer $k \geq 1$, with the $\bmod$ function taking values in the set $\{1,2, \ldots, N\}$; that is, if $k=$ $j N+q$ for some integers $j \geq 0$ and $0 \leq q<N$, then $T_{[k]}=T_{N}$ if $q=0$ and $T_{[k]}=T_{q}$ if $1 \leq q<N$.

The following convergence analysis was presented in [11].

Theorem ZWY ([11, Theorem 3.2]). Let $\mu \in\left(0,2 \beta / L^{2}\right)$, $\left\{\alpha_{n}\right\}_{n=0}^{\infty} \subset(0,1]$, and $\left\{\rho_{n}\right\}_{n=0}^{\infty} \subset(0,2 \alpha]$, such that (i) $\lim _{n \rightarrow \infty} \alpha_{n}=0$, (ii) $\sum_{n=0}^{\infty} \alpha_{n}=\infty$, (iii) $\lim _{n \rightarrow \infty}\left(\alpha_{n}-\right.$ $\left.\alpha_{n+N}\right) / \alpha_{n+N}=0$ or $\sum_{n=0}^{\infty}\left|\alpha_{n+N}-\alpha_{n}\right|<\infty$, (iv) $\lim _{n \rightarrow \infty}\left(\rho_{n}-\right.$ $\left.\rho_{n+N}\right) / \rho_{n+N}=0$ or $\sum_{n=0}^{\infty}\left|\rho_{n+N}-\rho_{n}\right|<\infty$, and (v) $\rho_{n} \leq \alpha_{n}$ for all $n \geq 0$. Assume in addition that

$$
\begin{aligned}
\bigcap_{i=1}^{N} \operatorname{Fix}\left(T_{i}\right) & =\operatorname{Fix}\left(T_{1} T_{2} \cdots T_{N}\right) \\
& =\operatorname{Fix}\left(T_{N} T_{1} \cdots T_{N-1}\right) \\
& =\cdots=\operatorname{Fix}\left(T_{2} T_{3} \cdots T_{N} T_{1}\right) .
\end{aligned}
$$

Then, the sequence $\left\{x_{n}\right\}_{n=0}^{\infty}$ generated by Algorithm ZWY satisfies the following properties:

(a) $\left\{x_{n}\right\}_{n=0}^{\infty}$ is bounded;

(b) $\lim _{n \rightarrow \infty}\left\|x_{n+N}-x_{n}\right\|=0$ and $\lim _{n \rightarrow \infty} \| x_{n}-$ $T_{n+N} \cdots T_{n+1} x_{n} \|=0$

(c) $\left\{x_{n}\right\}_{n=0}^{\infty}$ converges strongly to the unique solution of Problem I provided $\left\|x_{n}-y_{n}\right\|=o\left(\rho_{n}\right)$.

In this paper, we introduce and study the following triple hierarchical variational inequality (THVI) with constraints of finitely many GMEPs, finitely many variational inclusions, and general system of variational inequalities.

Problem II. Let $M, N$ be two integers. Assume that

(i) $\left\{T_{n}\right\}_{n=1}^{\infty}$ is a sequence of nonexpansive self-mappings on $C$ and $F_{j}: C \rightarrow H$ is $\zeta_{j}$-inverse strongly monotone for $j=1,2$;

(ii) $\widetilde{A}_{1}: H \rightarrow H$ is $\alpha$-inverse strongly monotone and $\widetilde{A}_{2}: H \rightarrow H$ is $\beta$-strongly monotone and $L$ Lipschitz continuous;

(iii) $\Theta_{k}$ is a bifunction from $C \times C$ to $\mathbf{R}$ satisfying (A1)(A4) and $\varphi_{k}: C \rightarrow \mathbf{R} \cup\{+\infty\}$ is a proper lower semicontinuous and convex function, where $k \in$ $\{1,2, \ldots, M\}$ 
(iv) $R_{i}: C \rightarrow 2^{H}$ is a maximal monotone mapping, and $A_{k}: H \rightarrow H$ and $B_{i}: C \rightarrow H$ are $\mu_{k}{ }^{-}$ inverse strongly monotone and $\eta_{i}$-inverse strongly monotone, respectively, where $k \in\{1,2, \ldots, M\}$ and $i \in\{1,2, \ldots, N\}$;

(v) $\operatorname{VI}\left(\Omega, \widetilde{A_{1}}\right) \neq \emptyset$, where $\Omega:=\bigcap_{n=1}^{\infty} \operatorname{Fix}\left(T_{n}\right) \cap$ $\bigcap_{k=1}^{M} \operatorname{GMEP}\left(\Theta_{k}, \varphi_{k}, A_{k}\right) \cap \bigcap_{i=1}^{N} I\left(B_{i}, R_{i}\right) \cap \operatorname{GSVI}(G)$.

Then, the objective is to

$$
\begin{aligned}
& \text { find } x^{*} \in \operatorname{VI}\left(\operatorname{VI}\left(\Omega, \widetilde{A}_{1}\right), \widetilde{A}_{2}\right) \\
& :=\left\{x^{*} \in \mathrm{VI}\left(\Omega, \widetilde{A_{1}}\right):\left\langle\widetilde{A}_{2} x^{*}, v-x^{*}\right\rangle \geq 0,\right. \\
& \left.\forall v \in \operatorname{VI}\left(\Omega, \widetilde{A}_{1}\right)\right\} .
\end{aligned}
$$

Motivated and inspired by the above facts, we introduce and analyze a relaxed iterative algorithm by combining Korpelevich's extragradient method, hybrid steepest-descent method, and Mann's iteration method. We prove that under mild conditions, the proposed algorithm converges strongly to a common element $x^{*} \in \Omega:=\bigcap_{n=1}^{\infty} \operatorname{Fix}\left(T_{n}\right) \cap$ $\bigcap_{k=1}^{M} \operatorname{GMEP}\left(\Theta_{k}, \varphi_{k}, A_{k}\right) \cap \bigcap_{i=1}^{N} I\left(B_{i}, R_{i}\right) \cap \operatorname{GSVI}(G)$ of the fixed point set of infinitely many nonexpansive mappings $\left\{T_{n}\right\}_{n=1}^{\infty}$, the solution set of finitely many GMEPs, the solution set of finitely many variational inclusions, and the solution set of GSVI (10), which is just a unique solution of the THVI (19). In addition, we also consider the application of the proposed algorithm to solve a hierarchical variational inequality problem with constraints of finitely many GMEPs, finitely many variational inclusions, and GSVI (10). That is, under appropriate conditions, we prove that the proposed algorithm converges strongly to a unique solution $u^{*} \in \Omega$ of the VIP: $\left\langle\widetilde{A}_{2} u^{*}, p-u^{*}\right\rangle \geq 0, \forall p \in \Omega$; equivalently, $P_{\Omega}\left(I-\widetilde{A_{2}}\right) u^{*}=u^{*}$. The results obtained in this paper improve and extend the corresponding results announced by many others including $[12,13]$. A comprehensive survey on triple hierarchical variational inequalities can be found in [14].

\section{Preliminaries}

Throughout this paper, we assume that $H$ is a real Hilbert space whose inner product and norm are denoted by $\langle\cdot, \cdot\rangle$ and $\|\cdot\|$, respectively. Let $C$ be a nonempty closed convex subset of $H$. We write $x_{n} \rightarrow x$ to indicate that the sequence $\left\{x_{n}\right\}$ converges weakly to $x$ and $x_{n} \rightarrow x$ to indicate that the sequence $\left\{x_{n}\right\}$ converges strongly to $x$. Moreover, we use $\omega_{w}\left(x_{n}\right)$ to denote the weak $\omega$-limit set of the sequence $\left\{x_{n}\right\}$; that is,

$$
\begin{aligned}
\omega_{w}\left(x_{n}\right):= & \left\{x \in H: x_{n_{i}} \rightarrow x\right. \\
& \text { for some subsequence } \left.\left\{x_{n_{i}}\right\} \text { of }\left\{x_{n}\right\}\right\} .
\end{aligned}
$$

Recall that a mapping $A: C \rightarrow H$ is called

(i) monotone if

$$
\langle A x-A y, x-y\rangle \geq 0, \quad \forall x, y \in C ;
$$

(ii) $\eta$-strongly monotone if there exists a constant $\eta>0$ such that

$$
\langle A x-A y, x-y\rangle \geq \eta\|x-y\|^{2}, \quad \forall x, y \in C ;
$$

(iii) $\alpha$-inverse strongly monotone if there exists a constant $\alpha>0$ such that

$$
\langle A x-A y, x-y\rangle \geq \alpha\|A x-A y\|^{2}, \quad \forall x, y \in C .
$$

It is obvious that if $A$ is $\alpha$-inverse strongly monotone, then $A$ is monotone and $(1 / \alpha)$-Lipschitz continuous. Moreover, we also have that, for all $u, v \in C$ and $\lambda>0$,

$$
\begin{aligned}
\|(I- & \lambda A) u-(I-\lambda A) v \|^{2} \\
& =\|(u-v)-\lambda(A u-A v)\|^{2} \\
& =\|u-v\|^{2}-2 \lambda\langle A u-A v, u-v\rangle+\lambda^{2}\|A u-A v\|^{2} \\
& \leq\|u-v\|^{2}+\lambda(\lambda-2 \alpha)\|A u-A v\|^{2} .
\end{aligned}
$$

So, if $\lambda \leq 2 \alpha$, then $I-\lambda A$ is a nonexpansive mapping from $C$ to $H$.

The metric (or nearest point) projection from $H$ onto $C$ is the mapping $P_{C}: H \rightarrow C$ which assigns to each point $x \in H$ the unique point $P_{C} x \in C$, satisfying the property

$$
\left\|x-P_{C} x\right\|=\inf _{y \in C}\|x-y\|=: d(x, C) .
$$

Some important properties of projections are gathered in the following proposition.

Proposition 1. For given $x \in H$ and $z \in C$ :

(i) $z=P_{C} x \Leftrightarrow\langle x-z, y-z\rangle \leq 0, \forall y \in C$;

(ii) $z=P_{C} x \Leftrightarrow\|x-z\|^{2} \leq\|x-y\|^{2}-\|y-z\|^{2}, \forall y \in C$;

(iii) $\left\langle P_{C} x-P_{C} y, x-y\right\rangle \geq\left\|P_{C} x-P_{C} y\right\|^{2}, \forall y \in H$.

Consequently, $P_{C}$ is nonexpansive and monotone.

Definition 2. A mapping $T: H \rightarrow H$ is said to be

(a) nonexpansive if

$$
\|T x-T y\| \leq\|x-y\|, \quad \forall x, y \in H
$$

(b) firmly nonexpansive if $2 T-I$ is nonexpansive or, equivalently, if $T$ is 1 -inverse strongly monotone (1-ism),

$$
\langle x-y, T x-T y\rangle \geq\|T x-T y\|^{2}, \quad \forall x, y \in H
$$

alternatively, $T$ is firmly nonexpansive if and only if $T$ can be expressed as

$$
T=\frac{1}{2}(I+S),
$$

where $S: H \rightarrow H$ is nonexpansive; projections are firmly nonexpansive. 
It can be easily seen that if $T$ is nonexpansive, then $I-T$ is monotone. It is also easy to see that a projection $P_{C}$ is 1-ism. Inverse strongly monotone (also referred to as cocoercive) operators have been applied widely in solving practical problems in various fields.

Next, we list some elementary conclusions for the mixed equilibrium problem $(\mathrm{MEP})$ where $\operatorname{MEP}(\Theta, \varphi)$ is the solution set.

Proposition 3 (see [15]). Assume that $\Theta: C \times C \rightarrow \mathbf{R}$ satisfies (A1)-(A4) and let $\varphi: C \rightarrow \mathbf{R}$ be a proper lower semicontinuous and convex function. Assume that either (B1) or (B2) holds. For $r>0$ and $x \in H$, define a mapping $T_{r}^{(\Theta, \varphi)}: H \rightarrow C$ as follows:

$$
\begin{array}{r}
T_{r}^{(\Theta, \varphi)}(x)=\{z \in C: \Theta(z, y)+\varphi(y)-\varphi(z) \\
\left.+\frac{1}{r}\langle y-z, z-x\rangle \geq 0, \forall y \in C\right\}
\end{array}
$$

for all $x \in H$. Then, the following hold:

(i) for each $x \in H, T_{r}^{(\Theta, \varphi)}(x)$ is nonempty and singlevalued;

(ii) $T_{r}^{(\Theta, \varphi)}$ is firmly nonexpansive; that is, for any $x, y \in H$,

$$
\left\|T_{r}^{(\Theta, \varphi)} x-T_{r}^{(\Theta, \varphi)} y\right\|^{2} \leq\left\langle T_{r}^{(\Theta, \varphi)} x-T_{r}^{(\Theta, \varphi)} y, x-y\right\rangle ;
$$

(iii) $\operatorname{Fix}\left(T_{r}^{(\Theta, \varphi)}\right)=\operatorname{MEP}(\Theta, \varphi)$;

(iv) $\operatorname{MEP}(\Theta, \varphi)$ is closed and convex;

(v) $\left\|T_{s}^{(\Theta, \varphi)} x-T_{t}^{(\Theta, \varphi)} x\right\|^{2} \leq((s-t) / s)\left\langle T_{s}^{(\Theta, \varphi)} x-T_{t}^{(\Theta, \varphi)} x\right.$, $\left.T_{s}^{(\Theta, \varphi)} x-x\right\rangle$ for all $s, t>0$ and $x \in H$.

We need some facts and tools in a real Hilbert space $H$ which are listed as lemmas below.

Lemma 4. Let $X$ be a real inner product space. Then, there holds the following inequality:

$$
\|x+y\|^{2} \leq\|x\|^{2}+2\langle y, x+y\rangle, \quad \forall x, y \in X .
$$

Lemma 5. Let $A: C \rightarrow H$ be a monotone mapping. In the context of the variational inequality problem, the characterization of the projection (see Proposition 1(i)) implies

$$
u \in \mathrm{VI}(C, A) \Longleftrightarrow u=P_{C}(u-\lambda A u), \quad \lambda>0 .
$$

Lemma 6 (see [16, demiclosedness principle]). Let $C$ be a nonempty closed convex subset of a real Hilbert space H. Let $T$ be a nonexpansive self-mapping on $C$. Then, $I-T$ is demiclosed. That is, whenever $\left\{x_{n}\right\}$ is a sequence in $C$ weakly converging to some $x \in C$ and the sequence $\left\{(I-T) x_{n}\right\}$ strongly converges to some $y$, it follows that $(I-T) x=y$. Here, $I$ is the identity operator of $H$.

Let $\left\{T_{n}\right\}_{n=1}^{\infty}$ be an infinite family of nonexpansive selfmappings on $C$ and let $\left\{\lambda_{n}\right\}_{n=1}^{\infty}$ be a sequence of nonnegative numbers in $[0,1]$. For any $n \geq 0$, define a mapping $W_{n}$ on $C$ as follows:

$$
\begin{aligned}
& U_{n, n+1}=I, \\
& U_{n, n}=\lambda_{n} T_{n} U_{n, n+1}+\left(1-\lambda_{n}\right) I, \\
& U_{n, n-1}=\lambda_{n-1} T_{n-1} U_{n, n}+\left(1-\lambda_{n-1}\right) I, \\
& \vdots \\
& U_{n, k}=\lambda_{k} T_{k} U_{n, k+1}+\left(1-\lambda_{k}\right) I, \\
& U_{n, k-1}=\lambda_{k-1} T_{k-1} U_{n, k}+\left(1-\lambda_{k-1}\right) I, \\
& \vdots \\
& U_{n, 2}=\lambda_{2} T_{2} U_{n, 3}+\left(1-\lambda_{2}\right) I, \\
& W_{n}=U_{n, 1}=\lambda_{1} T_{1} U_{n, 2}+\left(1-\lambda_{1}\right) I .
\end{aligned}
$$

Such a mapping $W_{n}$ is called the $W$-mapping generated by $T_{n}, T_{n-1}, \ldots, T_{1}$ and $\lambda_{n}, \lambda_{n-1}, \ldots, \lambda_{1}$.

Lemma 7 (see [17, Lemma 3.2]). Let $C$ be a nonempty closed convex subset of a real Hilbert space $H$. Let $\left\{T_{n}\right\}_{n=1}^{\infty}$ be a sequence of nonexpansive self-mappings on $C$ such that $\bigcap_{n=1}^{\infty} \operatorname{Fix}\left(T_{n}\right) \neq \emptyset$ and let $\left\{\lambda_{n}\right\}_{n=1}^{\infty}$ be a sequence in $(0, b]$ for some $b \in(0,1)$. Then, for every $x \in C$ and $k \geq 1$, the limit $\lim _{n \rightarrow \infty} U_{n, k} x$ exists where $U_{n, k}$ is defined as in (33).

Lemma 8 (see [17, Lemma 3.3]). Let $C$ be a nonempty closed convex subset of a real Hilbert space $H$. Let $\left\{T_{n}\right\}_{n=1}^{\infty}$ be a sequence of nonexpansive self-mappings on $C$ such that $\bigcap_{n=1}^{\infty} \operatorname{Fix}\left(T_{n}\right) \neq \emptyset$, and let $\left\{\lambda_{n}\right\}_{n=1}^{\infty}$ be a sequence in $(0, b]$ for some $b \in(0,1)$. Then, $\operatorname{Fix}(W)=\bigcap_{n=1}^{\infty} \operatorname{Fix}\left(T_{n}\right)$.

Let $C$ be a nonempty closed convex subset of a real Hilbert space $H$. We introduce some notations. Let $\lambda$ be a number in $(0,1]$ and let $\mu>0$. Associating with a nonexpansive mapping $T: C \rightarrow H$, we define the mapping $T^{\lambda}: C \rightarrow H$ by

$$
T^{\lambda} x:=T x-\lambda \mu F(T x), \quad \forall x \in C,
$$

where $F: H \rightarrow H$ is an operator such that, for some positive constants $\kappa, \eta>0, F$ is $\kappa$-Lipschitzian and $\eta$-strongly monotone on $H$; that is, $F$ satisfies the conditions:

$$
\begin{gathered}
\|F x-F y\| \leq \kappa\|x-y\|, \\
\langle F x-F y, x-y\rangle \geq \eta\|x-y\|^{2},
\end{gathered}
$$

for all $x, y \in H$.

Lemma 9 (see [18, Lemma 3.1]). $T^{\lambda}$ is a contraction provided $0<\mu<2 \eta / \kappa^{2}$; that is,

$$
\left\|T^{\lambda} x-T^{\lambda} y\right\| \leq(1-\lambda \tau)\|x-y\|, \quad \forall x, y \in C,
$$

where $\tau=1-\sqrt{1-\mu\left(2 \eta-\mu \kappa^{2}\right)} \in(0,1]$. 
Lemma 10 (see [18]). Let $\left\{s_{n}\right\}$ be a sequence of nonnegative numbers satisfying the conditions

$$
s_{n+1} \leq\left(1-\alpha_{n}\right) s_{n}+\alpha_{n} \beta_{n}, \quad \forall n \geq 1,
$$

where $\left\{\alpha_{n}\right\}$ and $\left\{\beta_{n}\right\}$ are sequences of real numbers such that

(i) $\left\{\alpha_{n}\right\} \subset[0,1]$ and $\sum_{n=1}^{\infty} \alpha_{n}=\infty$ or, equivalently,

$$
\prod_{n=1}^{\infty}\left(1-\alpha_{n}\right):=\lim _{n \rightarrow \infty} \prod_{k=1}^{n}\left(1-\alpha_{k}\right)=0 ;
$$

(ii) $\lim \sup _{n \rightarrow \infty} \beta_{n} \leq 0$, or $\sum_{n=1}^{\infty}\left|\alpha_{n} \beta_{n}\right|<\infty$.

Then, $\lim _{n \rightarrow \infty} s_{n}=0$.

Lemma 11 (see [16]). Let $H$ be a real Hilbert space. Then, the following hold:

(a) $\|x-y\|^{2}=\|x\|^{2}-\|y\|^{2}-2\langle x-y, y\rangle$ for all $x, y \in H$;

(b) $\|\lambda x+\mu y\|^{2}=\lambda\|x\|^{2}+\mu\|y\|^{2}-\lambda \mu\|x-y\|^{2}$ for all $x, y \in H$ and $\lambda, \mu \in[0,1]$ with $\lambda+\mu=1$;

(c) If $\left\{x_{n}\right\}$ is a sequence in $H$ such that $x_{n} \rightarrow x$, it follows that

$$
\begin{aligned}
& \limsup _{n \rightarrow \infty}\left\|x_{n}-y\right\|^{2} \\
& \quad=\limsup _{n \rightarrow \infty}\left\|x_{n}-x\right\|^{2}+\|x-y\|^{2}, \quad \forall y \in H .
\end{aligned}
$$

Finally, recall that a set-valued mapping $T: D(T) \subset H \rightarrow$ $2^{H}$ is called monotone if, for all $x, y \in D(T), f \in T x$ and $g \in T y$ imply

$$
\langle f-g, x-y\rangle \geq 0 \text {. }
$$

A set-valued mapping $T$ is called maximal monotone if $T$ is monotone and $(I+\lambda T) D(T)=H$ for each $\lambda>0$, where $I$ is the identity mapping of $H$. We denote by $G(T)$ the graph of $T$. It is known that a monotone mapping $T$ is maximal if and only if, for $(x, f) \in H \times H,\langle f-g, x-y\rangle \geq 0$ for every $(y, g) \in G(T)$ implies $f \in T x$. Let $A: C \rightarrow H$ be a monotone, $k$-Lipschitzcontinuous mapping and let $N_{C} v$ be the normal cone to $C$ at $v \in C$; that is,

$$
N_{C} v=\{u \in H:\langle v-p, u\rangle \geq 0, \forall p \in C\} .
$$

Define

$$
\widetilde{T} v= \begin{cases}A v+N_{C} v, & \text { if } v \in C, \\ \emptyset, & \text { if } v \notin C .\end{cases}
$$

Then, $\widetilde{T}$ is maximal monotone (see [19]) such that

$$
0 \in \widetilde{T} v \Longleftrightarrow v \in \operatorname{VI}(C, A) \text {. }
$$

Let $R: D(R) \subset H \rightarrow 2^{H}$ be a maximal monotone mapping. Let $\lambda, \mu>0$ be two positive numbers.
Lemma 12 (see [20]). There holds the resolvent identity

$$
J_{R, \lambda} x=J_{R, \mu}\left(\frac{\mu}{\lambda} x+\left(1-\frac{\mu}{\lambda}\right) J_{R, \lambda} x\right), \quad \forall x \in H .
$$

Remark 13. For $\lambda, \mu>0$, there holds the following relation:

$$
\begin{array}{r}
\left\|J_{R, \lambda} x-J_{R, \mu} y\right\| \\
\leq\|x-y\|+|\lambda-\mu|\left(\frac{1}{\lambda}\left\|J_{R, \lambda} x-y\right\|+\frac{1}{\mu}\left\|x-J_{R, \mu} y\right\|\right), \\
\forall x, y \in H .
\end{array}
$$

In terms of Huang [21], there holds the following property for the resolvent operator $J_{R, \lambda}: H \rightarrow \overline{D(R)}$.

Lemma 14. $J_{R, \lambda}$ is single-valued and firmly nonexpansive; that is,

$$
\left\langle J_{R, \lambda} x-J_{R, \lambda} y, x-y\right\rangle \geq\left\|J_{R, \lambda} x-J_{R, \lambda} y\right\|^{2}, \quad \forall x, y \in H .
$$

Consequently, $J_{R, \lambda}$ is nonexpansive and monotone.

Lemma 15 (see [22]). Let $R$ be a maximal monotone mapping with $D(R)=C$. Then, for any given $\lambda>0, u \in C$ is a solution of problem (12) if and only if $u \in C$ satisfies

$$
u=J_{R, \lambda}(u-\lambda B u)
$$

Lemma 16 (see [23]). Let $R$ be a maximal monotone mapping with $D(R)=C$ and let $B: C \rightarrow H$ be a strongly monotone, continuous, and single-valued mapping. Then, for each $z \in H$, the equation $z \in(B+\lambda R) x$ has a unique solution $x_{\lambda}$ for $\lambda>0$.

Lemma 17 (see [22]). Let $R$ be a maximal monotone mapping with $D(R)=C$ and let $B: C \rightarrow H$ be a monotone, continuous, and single-valued mapping. Then, $(I+\lambda(R+B)) C=H$ for each $\lambda>0$. In this case, $R+B$ is maximal monotone.

\section{Main Results}

In this section, we will introduce and analyze a relaxed iterative algorithm for finding a solution of the THVI (19) with constraints of several problems: finitely many GMEPs, finitely many variational inclusions, and GSVI (10) in a real Hilbert space. This algorithm is based on Korpelevich's extragradient method, hybrid steepest-descent method, and Mann's iteration method. We prove the strong convergence of the proposed algorithm to a unique solution of THVI (19) under suitable conditions. In addition, we also consider the application of the proposed algorithm to solve a hierarchical VIP with the same constraints.

We are now in a position to state and prove our first main result.

Theorem 18. Let $C$ be a nonempty closed convex subset of a real Hilbert space $H$. Let $M, N$ be two integers. Let $\Theta_{k}$ be a bifunction from $C \times C$ to $\mathbf{R}$ satisfying (A1)-(A4) and let 
$\varphi_{k}: C \rightarrow \mathbf{R} \cup\{+\infty\}$ be a proper lower semicontinuous and convex function, where $k \in\{1,2, \ldots, M\}$. Let $R_{i}: C \rightarrow 2^{H}$ be a maximal monotone mapping and let $A_{k}: H \rightarrow H$ and $B_{i}: C \rightarrow H$ be $\mu_{k}$-inverse strongly monotone and $\eta_{i}$-inverse strongly monotone, respectively, where $k \in\{1,2, \ldots, M\}, i \in$ $\{1,2, \ldots, N\}$. Let $\left\{T_{n}\right\}_{n=1}^{\infty}$ be a sequence of nonexpansive selfmappings on $C$ and $\left\{\lambda_{n}\right\}_{n=1}^{\infty}$ be a sequence in $(0, b]$ for some $b \in(0,1)$. Let $F_{j}: C \rightarrow H$ be $\zeta_{j}$-inverse strongly monotone for $j=1,2$. Let $\widetilde{A}_{1}: H \rightarrow H$ be $\alpha$-inverse strongly monotone with $\left(I-\widetilde{A}_{1}\right) C \subset C$ and let $\widetilde{A}_{2}: H \rightarrow H$ be $\beta$-strongly monotone and L-Lipschitz continuous. Assume that either (B1) or (B2) holds and that $\operatorname{VI}\left(\Omega, \widetilde{A}_{1}\right) \neq \emptyset$ where $\Omega:=\bigcap_{n=1}^{\infty} \operatorname{Fix}\left(T_{n}\right) \cap \bigcap_{k=1}^{M} \operatorname{GMEP}\left(\Theta_{k}, \varphi_{k}, A_{k}\right) \cap \bigcap_{i=1}^{N} I\left(B_{i}, R_{i}\right) \cap$ $\operatorname{GSVI}(G)$. Let $\mu \in\left(0,2 \beta / L^{2}\right)$ and $\left\{\alpha_{n}\right\}_{n=1}^{\infty}$ and $\left\{\rho_{n}\right\}_{n=1}^{\infty}$ be sequences in $(0,1]$. For arbitrarily given $x_{1} \in H$, let $\left\{x_{n}\right\}$ be a sequence generated by

$$
\begin{aligned}
u_{n}= & T_{r_{M, n}}^{\left(\Theta_{M}, \varphi_{M}\right)}\left(I-r_{M, n} A_{M}\right) T_{r_{M-1, n}}^{\left(\Theta_{M-1}, \varphi_{M-1}\right)} \\
& \cdot\left(I-r_{M-1, n} A_{M-1}\right) \cdots T_{r_{1, n}}^{\left(\Theta_{1}, \varphi_{1}\right)}\left(I-r_{1, n} A_{1}\right) x_{n}, \\
v_{n}= & J_{R_{N}, \lambda_{N, n}}\left(I-\lambda_{N, n} B_{N}\right) J_{R_{N-1}, \lambda_{N-1, n}} \\
& \cdot\left(I-\lambda_{N-1, n} B_{N-1}\right) \cdots J_{R_{1}, \lambda_{1, n}}\left(I-\lambda_{1, n} B_{1}\right) u_{n}, \\
y_{n}= & W_{n} G\left(v_{n}-\rho_{n} \widetilde{A}_{1} v_{n}\right), \\
x_{n+1}= & y_{n}-\mu \alpha_{n} \widetilde{A}_{2} y_{n}, \quad \forall n \geq 1,
\end{aligned}
$$

where $G$ is defined as in (12) with $\nu_{j} \in\left(0,2 \zeta_{j}\right)$ for $j=1,2$, and $W_{n}$ is the $W$-mapping defined by (33). Suppose that the following conditions are satisfied:

(H1) $\lim _{n \rightarrow \infty} \alpha_{n}=0, \sum_{n=1}^{\infty} \alpha_{n}=\infty$ and $\lim _{n \rightarrow \infty}\left(1 / \alpha_{n}\right) \mid 1-$ $\rho_{n-1} / \rho_{n} \mid=0$;

(H2) $\lim _{n \rightarrow \infty}\left(1 / \alpha_{n}\right)\left|1 / \rho_{n}-1 / \rho_{n-1}\right|=0$ and $\lim _{n \rightarrow \infty}(1 /$ $\left.\rho_{n}\right)\left|1-\alpha_{n-1} / \alpha_{n}\right|=0$;

(H3) $\lim _{n \rightarrow \infty}\left(\rho_{n} / \alpha_{n}\right)=0$ and $\lim _{n \rightarrow \infty}\left(b^{n} / \alpha_{n} \rho_{n}\right)=0$;

(H4) $\left\{\lambda_{i, n}\right\} \subset\left[a_{i}, b_{i}\right] \subset\left(0,2 \eta_{i}\right)$ and $\lim _{n \rightarrow \infty}\left(\mid \lambda_{i, n}-\right.$ $\left.\lambda_{i, n-1} \mid / \alpha_{n} \rho_{n}\right)=0$ for all $i \in\{1,2, \ldots, N\}$;

(H5) $\left\{r_{k, n}\right\} \subset\left[e_{k}, f_{k}\right] \subset\left(0,2 \mu_{k}\right)$ and $\lim _{n \rightarrow \infty}\left(\mid r_{k, n}-\right.$ $\left.r_{k, n-1} \mid / \rho_{n} \alpha_{n}\right)=0$ for all $k \in\{1,2, \ldots, M\}$.

Then, there hold the following:

(i) $\lim _{n \rightarrow \infty}\left(\left\|x_{n+1}-x_{n}\right\| / \rho_{n}\right)=0$;

(ii) $\omega_{w}\left(x_{n}\right) \subset \Omega$;

(iii) $\omega_{w}\left(x_{n}\right) \subset \operatorname{VI}\left(\Omega, \widetilde{A}_{1}\right)$ provided $\left\|x_{n}-y_{n}\right\|=o\left(\rho_{n}\right)$ additionally.

Proof. Let $\left\{x^{*}\right\}=\operatorname{VI}\left(\operatorname{VI}\left(\Omega, \widetilde{A}_{1}\right), \widetilde{A}_{2}\right)$. Taking into account that $\lim _{n \rightarrow \infty}\left(\rho_{n} / \alpha_{n}\right)=0$, we may assume, without loss of generality, that $\rho_{n} \leq \alpha_{n}$ and $\rho_{n} \leq 2 \alpha$ for all $n \geq 1$. Since $\widetilde{A}_{2}$ is $L$-Lipschitz continuous, we get

$$
\left\|\widetilde{A}_{2} y_{n}-\widetilde{A}_{2} x^{*}\right\| \leq L\left\|y_{n}-x^{*}\right\|, \quad \forall n \geq 1 .
$$

Putting $\widetilde{v}_{n}=v_{n}-\rho_{n} \widetilde{A}_{1} v_{n}$ for all $n \geq 1$, we have

$$
\begin{aligned}
x_{n+1} & =y_{n}-\mu \alpha_{n} \widetilde{A}_{2} y_{n} \\
& =W_{n} G \widetilde{v}_{n}-\mu \alpha_{n} \widetilde{A}_{2} W_{n} G \widetilde{v}_{n} \\
& =\left(I-\mu \alpha_{n} \widetilde{A}_{2}\right) W_{n} G \widetilde{v}_{n}, \quad \forall n \geq 1 .
\end{aligned}
$$

Moreover, observe that

$$
\widetilde{v}_{n}=v_{n}-\rho_{n} \widetilde{A}_{1} v_{n}=\left(1-\rho_{n}\right) v_{n}+\rho_{n}\left(I-\widetilde{A}_{1}\right) v_{n} .
$$

(This is Mann's iteration method.) Since $\left(I-\widetilde{A}_{1}\right) C \subset C$ and $\left\{v_{n}\right\}_{n=1}^{\infty} \subset C$, we know from the convexity of $C$ that $\left\{\widetilde{v}_{n}\right\}_{n=1}^{\infty} \subset$ C. Put

$$
\begin{aligned}
\Delta_{n}^{k}= & T_{r_{k, n}}^{\left(\Theta_{k}, \varphi_{k}\right)}\left(I-r_{k, n} A_{k}\right) T_{r_{k-1, n}}^{\left(\Theta_{k-1}, \varphi_{k-1}\right)} \\
& \cdot\left(I-r_{k-1, n} A_{k-1}\right) \cdots T_{r_{1, n}}^{\left(\Theta_{1}, \varphi_{1}\right)}\left(I-r_{1, n} A_{1}\right) x_{n}
\end{aligned}
$$

for all $k \in\{1,2, \ldots, M\}$ and $n \geq 1$,

$$
\begin{aligned}
\Lambda_{n}^{i}= & J_{R_{i}, \lambda_{i, n}}\left(I-\lambda_{i, n} B_{i}\right) J_{R_{i-1}, \lambda_{i-1, n}} \\
& \cdot\left(I-\lambda_{i-1, n} B_{i-1}\right) \cdots J_{R_{1}, \lambda_{1, n}}\left(I-\lambda_{1, n} B_{1}\right)
\end{aligned}
$$

for all $i \in\{1,2, \ldots, N\}, \Delta_{n}^{0}=I$, and $\Lambda_{n}^{0}=I$, where $I$ is the identity mapping on $H$. Then, we have that $u_{n}=\Delta_{n}^{M} x_{n}$ and $v_{n}=\Lambda_{n}^{N} u_{n}$.

We divide the rest of the proof into several steps.

Step 1. We prove that $\left\{x_{n}\right\}$ is bounded.

Indeed, utilizing (24) and Proposition 3(ii), we have

$$
\begin{aligned}
\left\|u_{n}-x^{*}\right\|= & \| T_{r_{M, n}^{\left(\Theta_{M}, \varphi_{M}\right)}}^{\left(I-r_{M, n} B_{M}\right) \Delta_{n}^{M-1} x_{n}} \\
& -T_{r_{M, n}}^{\left(\Theta_{M}, \varphi_{M}\right)}\left(I-r_{M, n} B_{M}\right) \Delta_{n}^{M-1} x^{*} \| \\
\leq & \left\|\left(I-r_{M, n} B_{M}\right) \Delta_{n}^{M-1} x_{n}-\left(I-r_{M, n} B_{M}\right) \Delta_{n}^{M-1} x^{*}\right\| \\
\leq & \left\|\Delta_{n}^{M-1} x_{n}-\Delta_{n}^{M-1} x^{*}\right\| \\
\vdots & \\
\leq & \left\|\Delta_{n}^{0} x_{n}-\Delta_{n}^{0} x^{*}\right\| \\
= & \left\|x_{n}-x^{*}\right\| .
\end{aligned}
$$


Utilizing (24) and Lemma 14, we have

$$
\begin{aligned}
\left\|v_{n}-x^{*}\right\|= & \| J_{R_{N}, \lambda_{N, n}}\left(I-\lambda_{N, n} A_{N}\right) \Lambda_{n}^{N-1} u_{n} \\
& \quad-J_{R_{N}, \lambda_{N, n}}\left(I-\lambda_{N, n} A_{N}\right) \Lambda_{n}^{N-1} x^{*} \| \\
\leq & \left\|\left(I-\lambda_{N, n} A_{N}\right) \Lambda_{n}^{N-1} u_{n}-\left(I-\lambda_{N, n} A_{N}\right) \Lambda_{n}^{N-1} x^{*}\right\| \\
\leq & \left\|\Lambda_{n}^{N-1} u_{n}-\Lambda_{n}^{N-1} x^{*}\right\| \\
\vdots & \\
\leq & \left\|\Lambda_{n}^{0} u_{n}-\Lambda_{n}^{0} x^{*}\right\| \\
= & \left\|u_{n}-x^{*}\right\| .
\end{aligned}
$$

Combining (54) and (55), we have

$$
\left\|v_{n}-x^{*}\right\| \leq\left\|x_{n}-x^{*}\right\|
$$

Since $x^{*}=G x^{*}=P_{C}\left(I-v_{1} F_{1}\right) P_{C}\left(I-v_{2} F_{2}\right) x^{*}, F_{j}$ is $\zeta_{j}$-inverse strongly monotone for $j=1,2$, and $0 \leq v_{j} \leq 2 \zeta_{j}$ for $j=1,2$, we deduce that, for any $n \geq 1$,

$$
\begin{aligned}
\| G \widetilde{v}_{n}- & x^{*} \|^{2} \\
= & \| P_{C}\left(I-v_{1} F_{1}\right) P_{C}\left(I-v_{2} F_{2}\right) \widetilde{v}_{n} \\
& \quad-P_{C}\left(I-v_{1} F_{1}\right) P_{C}\left(I-v_{2} F_{2}\right) x^{*} \|^{2} \\
\leq & \|\left(I-v_{1} F_{1}\right) P_{C}\left(I-v_{2} F_{2}\right) \widetilde{v}_{n} \\
& \quad-\left(I-v_{1} F_{1}\right) P_{C}\left(I-v_{2} F_{2}\right) x^{*} \|^{2} \\
= & \|\left[P_{C}\left(I-v_{2} F_{2}\right) \widetilde{v}_{n}-P_{C}\left(I-v_{2} F_{2}\right) x^{*}\right] \\
& \quad-v_{1}\left[F_{1} P_{C}\left(I-v_{2} F_{2}\right) v_{n}-F_{1} P_{C}\left(I-v_{2} F_{2}\right) x^{*}\right] \|^{2} \\
\leq & \left\|P_{C}\left(I-v_{2} F_{2}\right) \widetilde{v}_{n}-P_{C}\left(I-v_{2} F_{2}\right) x^{*}\right\|^{2} \\
& +v_{1}\left(v_{1}-2 \zeta_{1}\right) \\
& \quad\left\|F_{1} P_{C}\left(I-v_{2} F_{2}\right) v_{n}-F_{1} P_{C}\left(I-v_{2} F_{2}\right) p\right\|^{2} \\
\leq & \left\|P_{C}\left(I-v_{2} F_{2}\right) \widetilde{v}_{n}-P_{C}\left(I-v_{2} F_{2}\right) x^{*}\right\|^{2} \\
\leq & \left\|\left(I-v_{2} F_{2}\right) \widetilde{v}_{n}-\left(I-v_{2} F_{2}\right) x^{*}\right\|^{2} \\
= & \left\|\left(\widetilde{v}_{n}-p\right)-v_{2}\left(F_{2} \widetilde{v}_{n}-F_{2} x^{*}\right)\right\|^{2} \\
\leq & \left\|\widetilde{v}_{n}-x^{*}\right\|^{2}+v_{2}\left(v_{2}-2 \zeta_{2}\right)\left\|F_{2} \widetilde{v}_{n}-F_{2} x^{*}\right\|^{2} \\
& \\
&
\end{aligned}
$$

Since $\widetilde{A}_{1}$ is $\alpha$-inverse strongly monotone and $\left\{\rho_{n}\right\}_{n=1}^{\infty} \subset$ $(0,2 \alpha]$, we have

$$
\begin{aligned}
& \left\|v_{n}-x^{*}-\rho_{n}\left(\widetilde{A}_{1} v_{n}-\widetilde{A}_{1} x^{*}\right)\right\|^{2} \\
& =\left\|v_{n}-x^{*}\right\|^{2}-2 \rho_{n}\left\langle\widetilde{A}_{1} v_{n}-\widetilde{A}_{1} x^{*}, x_{n}-x^{*}\right\rangle \\
& \quad+\rho_{n}^{2}\left\|\widetilde{A}_{1} v_{n}-\widetilde{A}_{1} x^{*}\right\|^{2} \\
& \leq\left\|v_{n}-x^{*}\right\|^{2}-\rho_{n}\left(2 \alpha-\rho_{n}\right)\left\|\widetilde{A}_{1} v_{n}-\widetilde{A}_{1} x^{*}\right\|^{2} \\
& \leq\left\|v_{n}-x^{*}\right\|^{2} .
\end{aligned}
$$

Utilizing Lemma 9, the nonexpansivity of $W_{n}$ and the one of $G$ (due to Proposition CWY), we obtain from (24) and (56) that

$$
\begin{aligned}
& \left\|x_{n+1}-x^{*}\right\| \\
& =\left\|\left(I-\mu \alpha_{n} \widetilde{A}_{2}\right) W_{n} G \widetilde{v}_{n}-x^{*}\right\| \\
& \leq\left\|\left(I-\mu \alpha_{n} \widetilde{A}_{2}\right) W_{n} G \widetilde{v}_{n}-\left(I-\mu \alpha_{n} \widetilde{A}_{2}\right) W_{n} G x^{*}\right\| \\
& +\left\|\left(I-\mu \alpha_{n} \widetilde{A_{2}}\right) W_{n} G x^{*}-W_{n} G x^{*}\right\| \\
& \leq\left(1-\alpha_{n} \tau\right)\left\|G \widetilde{v}_{n}-G x^{*}\right\|+\alpha_{n} \mu\left\|\widetilde{A}_{2} W_{n} G x^{*}\right\| \\
& \leq\left(1-\alpha_{n} \tau\right)\left\|\widetilde{v}_{n}-x^{*}\right\|+\alpha_{n} \mu\left\|\widetilde{A}_{2} x^{*}\right\| \\
& =\left(1-\alpha_{n} \tau\right)\left\|v_{n}-x^{*}-\rho_{n} \widetilde{A}_{1} v_{n}\right\|+\alpha_{n} \mu\left\|\widetilde{A}_{2} x^{*}\right\| \\
& =\left(1-\alpha_{n} \tau\right)\left\|v_{n}-x^{*}-\rho_{n}\left(\widetilde{A}_{1} v_{n}-\widetilde{A}_{1} x^{*}\right)-\rho_{n} \widetilde{A}_{1} x^{*}\right\| \\
& +\alpha_{n} \mu\left\|\widetilde{A_{2}} x^{*}\right\| \\
& \leq\left(1-\alpha_{n} \tau\right)\left[\left\|v_{n}-x^{*}-\rho_{n}\left(\widetilde{A}_{1} v_{n}-\widetilde{A}_{1} x^{*}\right)\right\|\right. \\
& \left.+\rho_{n}\left\|\widetilde{A}_{1} x^{*}\right\|\right]+\alpha_{n} \mu\left\|\widetilde{A}_{2} x^{*}\right\| \\
& \leq\left(1-\alpha_{n} \tau\right)\left[\left\|v_{n}-x^{*}\right\|+\rho_{n}\left\|\widetilde{A}_{1} x^{*}\right\|\right]+\alpha_{n} \mu\left\|\widetilde{A}_{2} x^{*}\right\| \\
& \leq\left(1-\alpha_{n} \tau\right)\left[\left\|x_{n}-x^{*}\right\|+\rho_{n}\left\|\widetilde{A_{1}} x^{*}\right\|\right]+\alpha_{n} \mu\left\|\widetilde{A_{2}} x^{*}\right\| \\
& \leq\left(1-\alpha_{n} \tau\right)\left\|x_{n}-x^{*}\right\|+\rho_{n}\left\|\widetilde{A}_{1} x^{*}\right\|+\alpha_{n} \mu\left\|\widetilde{A}_{2} x^{*}\right\| \\
& \leq\left(1-\alpha_{n} \tau\right)\left\|x_{n}-x^{*}\right\|+\alpha_{n}\left(\left\|\widetilde{A}_{1} x^{*}\right\|+\mu\left\|\widetilde{A}_{2} x^{*}\right\|\right) \\
& =\left(1-\alpha_{n} \tau\right)\left\|x_{n}-x^{*}\right\|+\alpha_{n} \tau \cdot \frac{\left\|\widetilde{A}_{1} x^{*}\right\|+\mu\left\|\widetilde{A}_{2} x^{*}\right\|}{\tau} \\
& \leq \max \left\{\left\|x_{n}-x^{*}\right\|, \frac{\left\|\widetilde{A}_{1} x^{*}\right\|+\mu\left\|\widetilde{A}_{2} x^{*}\right\|}{\tau}\right\} \text {, }
\end{aligned}
$$


where $\tau:=1-\sqrt{1-\mu\left(2 \beta-\mu L^{2}\right)}$. By induction, we find that

$$
\begin{aligned}
& \left\|x_{n+1}-x^{*}\right\| \\
& \quad \leq \max \left\{\left\|x_{1}-x^{*}\right\|, \frac{\left\|\widetilde{A}_{1} x^{*}\right\|+\mu\left\|\widetilde{A}_{2} x^{*}\right\|}{\tau}\right\}, \quad \forall n \geq 1 .
\end{aligned}
$$

Thus, $\left\{x_{n}\right\}_{n=1}^{\infty}$ is bounded and so are the sequences $\left\{u_{n}\right\}_{n=1}^{\infty}$, $\left\{v_{n}\right\}_{n=1}^{\infty},\left\{\widetilde{v}_{n}\right\}_{n=1}^{\infty}$, and $\left\{y_{n}\right\}_{n=1}^{\infty}$.

Step 2. We prove that $\lim _{n \rightarrow \infty}\left(\left\|x_{n+1}-x_{n}\right\| / \rho_{n}\right)=0$.

Indeed, utilizing (24) and (45), we obtain that

$$
\begin{aligned}
& \left\|v_{n+1}-v_{n}\right\| \\
& =\left\|\Lambda_{n+1}^{N} u_{n+1}-\Lambda_{n}^{N} u_{n}\right\| \\
& =\| J_{R_{N}, \lambda_{N, n+1}}\left(I-\lambda_{N, n+1} B_{N}\right) \Lambda_{n+1}^{N-1} u_{n+1} \\
& -J_{R_{N}, \lambda_{N, n}}\left(I-\lambda_{N, n} B_{N}\right) \Lambda_{n}^{N-1} u_{n} \| \\
& \leq \| J_{R_{N}, \lambda_{N, n+1}}\left(I-\lambda_{N, n+1} B_{N}\right) \Lambda_{n+1}^{N-1} u_{n+1} \\
& -J_{R_{N}, \lambda_{N, n+1}}\left(I-\lambda_{N, n} B_{N}\right) \Lambda_{n+1}^{N-1} u_{n+1} \| \\
& +\| J_{R_{N}, \lambda_{N, n+1}}\left(I-\lambda_{N, n} B_{N}\right) \Lambda_{n+1}^{N-1} u_{n+1} \\
& -J_{R_{N}, \lambda_{N, n}}\left(I-\lambda_{N, n} B_{N}\right) \Lambda_{n}^{N-1} u_{n} \| \\
& \leq \|\left(I-\lambda_{N, n+1} B_{N}\right) \Lambda_{n+1}^{N-1} u_{n+1} \\
& -\left(I-\lambda_{N, n} B_{N}\right) \Lambda_{n+1}^{N-1} u_{n+1} \| \\
& +\left\|\left(I-\lambda_{N, n} B_{N}\right) \Lambda_{n+1}^{N-1} u_{n+1}-\left(I-\lambda_{N, n} B_{N}\right) \Lambda_{n}^{N-1} u_{n}\right\| \\
& +\left|\lambda_{N, n+1}-\lambda_{N, n}\right| \\
& \times\left(\frac{1}{\lambda_{N, n+1}} \| J_{R_{N}, \lambda_{N, n+1}}\left(I-\lambda_{N, n} B_{N}\right) \Lambda_{n+1}^{N-1} u_{n+1}\right. \\
& -\left(I-\lambda_{N, n} B_{N}\right) \Lambda_{n}^{N-1} u_{n} \| \\
& +\frac{1}{\lambda_{N, n}} \|\left(I-\lambda_{N, n} B_{N}\right) \Lambda_{n+1}^{N-1} u_{n+1} \\
& \left.-J_{R_{N}, \lambda_{N, n}}\left(I-\lambda_{N, n} B_{N}\right) \Lambda_{n}^{N-1} u_{n} \|\right) \\
& \leq\left|\lambda_{N, n+1}-\lambda_{N, n}\right|\left(\left\|B_{N} \Lambda_{n+1}^{N-1} u_{n+1}\right\|+\widetilde{M}\right) \\
& +\left\|\Lambda_{n+1}^{N-1} u_{n+1}-\Lambda_{n}^{N-1} u_{n}\right\|
\end{aligned}
$$

$$
\begin{aligned}
\leq & \left|\lambda_{N, n+1}-\lambda_{N, n}\right|\left(\left\|B_{N} \Lambda_{n+1}^{N-1} u_{n+1}\right\|+\widetilde{M}\right) \\
& +\left|\lambda_{N-1, n+1}-\lambda_{N-1, n}\right|\left(\left\|B_{N-1} \Lambda_{n+1}^{N-2} u_{n+1}\right\|+\widetilde{M}\right) \\
& +\left\|\Lambda_{n+1}^{N-2} u_{n+1}-\Lambda_{n}^{N-2} u_{n}\right\|
\end{aligned}
$$

$$
\begin{aligned}
& \leq\left|\lambda_{N, n+1}-\lambda_{N, n}\right|\left(\left\|B_{N} \Lambda_{n+1}^{N-1} u_{n+1}\right\|+\widetilde{M}\right) \\
& \quad+\left|\lambda_{N-1, n+1}-\lambda_{N-1, n}\right|\left(\left\|B_{N-1} \Lambda_{n+1}^{N-2} u_{n+1}\right\|+\widetilde{M}\right) \\
& \quad+\cdots+\left|\lambda_{1, n+1}-\lambda_{1, n}\right|\left(\left\|B_{1} \Lambda_{n+1}^{0} u_{n+1}\right\|+\widetilde{M}\right) \\
& \quad+\left\|\Lambda_{n+1}^{0} u_{n+1}-\Lambda_{n}^{0} u_{n}\right\| \\
& \leq \widetilde{M}_{0} \sum_{i=1}^{N}\left|\lambda_{i, n+1}-\lambda_{i, n}\right|+\left\|u_{n+1}-u_{n}\right\|,
\end{aligned}
$$

where

$$
\begin{gathered}
\sup _{n \geq 1}\left\{\frac{1}{\lambda_{N, n+1}} \| J_{R_{N}, \lambda_{N, n+1}}\left(I-\lambda_{N, n} B_{N}\right) \Lambda_{n+1}^{N-1} u_{n+1}\right. \\
-\left(I-\lambda_{N, n} B_{N}\right) \Lambda_{n}^{N-1} u_{n} \| \\
+\frac{1}{\lambda_{N, n}} \|\left(I-\lambda_{N, n} B_{N}\right) \Lambda_{n+1}^{N-1} u_{n+1} \\
\left.-J_{R_{N}, \lambda_{N, n}}\left(I-\lambda_{N, n} B_{N}\right) \Lambda_{n}^{N-1} u_{n} \|\right\} \leq \widetilde{M},
\end{gathered}
$$

for some $\widetilde{M}>0$ and $\sup _{n \geq 1}\left\{\sum_{i=1}^{N}\left\|B_{i} \Lambda_{n+1}^{i-1} u_{n+1}\right\|+\widetilde{M}\right\} \leq \widetilde{M}_{0}$ for some $\widetilde{M}_{0}>0$. Hence, it follows from (24) and $\left\{\rho_{n}\right\}_{n=1}^{\infty} \subset$ $(0,2 \alpha]$ that

$$
\begin{aligned}
& \left\|\widetilde{v}_{n+1}-\widetilde{v}_{n}\right\| \\
& =\left\|\left(v_{n+1}-\rho_{n+1} \widetilde{A}_{1} v_{n+1}\right)-\left(v_{n}-\rho_{n} \widetilde{A}_{1} v_{n}\right)\right\| \\
& \leq\left\|\left(v_{n+1}-\rho_{n+1} \widetilde{A}_{1} v_{n+1}\right)-\left(v_{n}-\rho_{n+1} \widetilde{A}_{1} v_{n}\right)\right\| \\
& +\left\|\left(v_{n}-\rho_{n+1} \widetilde{A}_{1} v_{n}\right)-\left(v_{n}-\rho_{n} \widetilde{A}_{1} v_{n}\right)\right\| \\
& \leq\left\|v_{n+1}-v_{n}\right\|+\left|\rho_{n+1}-\rho_{n}\right|\left\|\widetilde{A}_{1} v_{n}\right\| \\
& \leq \widetilde{M}_{0} \sum_{i=1}^{N}\left|\lambda_{i, n+1}-\lambda_{i, n}\right|+\left\|u_{n+1}-u_{n}\right\| \\
& +\left|\rho_{n+1}-\rho_{n}\right|\left\|\widetilde{A}_{1} v_{n}\right\| .
\end{aligned}
$$


Also, utilizing Proposition 3(ii), (v), we deduce that

$$
\begin{aligned}
& \left\|u_{n+1}-u_{n}\right\| \\
& =\left\|\Delta_{n+1}^{M} x_{n+1}-\Delta_{n}^{M} x_{n}\right\|
\end{aligned}
$$

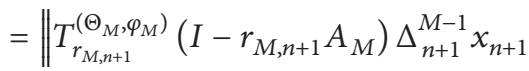

$$
\begin{aligned}
& -T_{r_{M, n}}^{\left(\Theta_{M}, \varphi_{M}\right)}\left(I-r_{M, n} A_{M}\right) \Delta_{n}^{M-1} x_{n} \| \\
& \leq \| T_{\left.r_{M, n+1}^{\left(\Theta_{M}, \varphi_{M}\right.}\right)}\left(I-r_{M, n+1} A_{M}\right) \Delta_{n+1}^{M-1} x_{n+1} \\
& -T_{r_{M, n}}^{\left(\Theta_{M}, \varphi_{M}\right)}\left(I-r_{M, n} A_{M}\right) \Delta_{n+1}^{M-1} x_{n+1} \| \\
& +\| T_{r_{M, n}}^{\left(\Theta_{M}, \varphi_{M}\right)}\left(I-r_{M, n} A_{M}\right) \Delta_{n+1}^{M-1} x_{n+1} \\
& -T_{r_{M, n}}^{\left(\Theta_{M}, \varphi_{M}\right)}\left(I-r_{M, n} A_{M}\right) \Delta_{n}^{M-1} x_{n} \|
\end{aligned}
$$

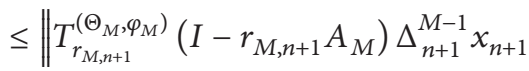

$$
\begin{aligned}
& -T_{r_{M, n}}^{\left(\Theta_{M}, \varphi_{M}\right)}\left(I-r_{M, n+1} A_{M}\right) \Delta_{n+1}^{M-1} x_{n+1} \| \\
& +\| T_{r_{M, n}}^{\left(\Theta_{M}, \varphi_{M}\right)}\left(I-r_{M, n+1} A_{M}\right) \Delta_{n+1}^{M-1} x_{n+1} \\
& -T_{r_{M, n}}^{\left(\Theta_{M}, \varphi_{M}\right)}\left(I-r_{M, n} A_{M}\right) \Delta_{n+1}^{M-1} x_{n+1} \| \\
& +\left\|\left(I-r_{M, n} A_{M}\right) \Delta_{n+1}^{M-1} x_{n+1}-\left(I-r_{M, n} A_{M}\right) \Delta_{n}^{M-1} x_{n}\right\| \\
& \leq \frac{\left|r_{M, n+1}-r_{M, n}\right|}{r_{M, n+1}} \| T_{r_{M, n+1}^{\left(\Theta_{M}, \varphi_{M}\right)}}\left(I-r_{M, n+1} A_{M}\right) \Delta_{n+1}^{M-1} x_{n+1} \\
& -\left(I-r_{M, n+1} A_{M}\right) \Delta_{n+1}^{M-1} x_{n+1} \| \\
& +\left|r_{M, n+1}-r_{M, n}\right|\left\|A_{M} \Delta_{n+1}^{M-1} x_{n+1}\right\| \\
& +\left\|\Delta_{n+1}^{M-1} x_{n+1}-\Delta_{n}^{M-1} x_{n}\right\| \\
& =\left|r_{M, n+1}-r_{M, n}\right| \\
& \cdot\left[\left\|A_{M} \Delta_{n+1}^{M-1} x_{n+1}\right\|\right.
\end{aligned}
$$

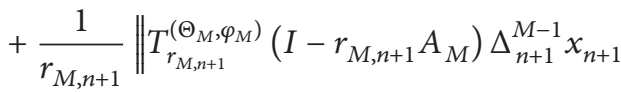

$$
\begin{aligned}
& \left.-\left(I-r_{M, n+1} A_{M}\right) \Delta_{n+1}^{M-1} x_{n+1} \|\right] \\
& +\left\|\Delta_{n+1}^{M-1} x_{n+1}-\Delta_{n}^{M-1} x_{n}\right\| \\
& \vdots \\
& \leq\left|r_{M, n+1}-r_{M, n}\right|
\end{aligned}
$$

$$
\begin{aligned}
& \cdot\left[\left\|A_{M} \Delta_{n+1}^{M-1} x_{n+1}\right\|\right.
\end{aligned}
$$

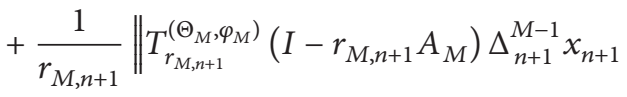

$$
\begin{aligned}
& \left.-\left(I-r_{M, n+1} A_{M}\right) \Delta_{n+1}^{M-1} x_{n+1} \|\right] \\
& +\cdots+\left|r_{1, n+1}-r_{1, n}\right| \\
& \cdot\left[\left\|A_{1} \Delta_{n+1}^{0} x_{n+1}\right\|\right. \\
& +\frac{1}{r_{1, n+1}} \| T_{r_{1, n+1}}^{\left(\Theta_{1}, \varphi_{1}\right)}\left(I-r_{1, n+1} A_{1}\right) \Delta_{n+1}^{0} x_{n+1} \\
& \left.-\left(I-r_{1, n+1} A_{1}\right) \Delta_{n+1}^{0} x_{n+1} \|\right] \\
& +\left\|\Delta_{n+1}^{0} x_{n+1}-\Delta_{n}^{0} x_{n}\right\| \\
& \leq \widetilde{M}_{1} \sum_{k=1}^{M}\left|r_{k, n+1}-r_{k, n}\right|+\left\|x_{n+1}-x_{n}\right\|,
\end{aligned}
$$

where $\widetilde{M}_{1}>0$ is a constant such that, for each $n \geq 1$,

$$
\begin{gathered}
\sum_{k=1}^{M}\left[\left\|A_{k} \Delta_{n+1}^{k-1} x_{n+1}\right\|+\frac{1}{r_{k, n+1}}\right. \\
\cdot \| T_{r_{k, n+1}^{\left(\Theta_{k}, \varphi_{k}\right)}\left(I-r_{k, n+1} A_{k}\right) \Delta_{n+1}^{k-1} x_{n+1}} \\
\left.\quad-\left(I-r_{k, n+1} A_{k}\right) \Delta_{n+1}^{k-1} x_{n+1} \|\right] \leq \widetilde{M}_{1} .
\end{gathered}
$$

In the meantime, from (33), since $W_{n}, T_{n}$, and $U_{n, i}$ are all nonexpansive, we have

$$
\begin{aligned}
&\left\|W_{n+1} G \widetilde{v}_{n}-W_{n} G \widetilde{v}_{n}\right\| \\
&=\left\|\lambda_{1} T_{1} U_{n+1,2} G \widetilde{v}_{n}-\lambda_{1} T_{1} U_{n, 2} G \widetilde{v}_{n}\right\| \\
& \leq \lambda_{1}\left\|U_{n+1,2} G \widetilde{v}_{n}-U_{n, 2} G \widetilde{v}_{n}\right\| \\
&= \lambda_{1}\left\|\lambda_{2} T_{2} U_{n+1,3} G \widetilde{v}_{n}-\lambda_{2} T_{2} U_{n, 3} G \widetilde{v}_{n}\right\| \\
& \leq \lambda_{1} \lambda_{2}\left\|U_{n+1,3} G \widetilde{v}_{n}-U_{n, 3} G \widetilde{v}_{n}\right\| \\
& \vdots \\
& \leq \lambda_{1} \lambda_{2} \cdots \lambda_{n}\left\|U_{n+1, n+1} G \widetilde{v}_{n}-U_{n, n+1} G \widetilde{v}_{n}\right\| \\
& \leq \widetilde{M}_{2} \prod_{i=1}^{n} \lambda_{i},
\end{aligned}
$$

where $\widetilde{M}_{2}$ is a constant such that $\left\|U_{n+1, n+1} G \widetilde{v}_{n}\right\|+$ $\left\|U_{n, n+1} G \widetilde{v}_{n}\right\| \leq \widetilde{M}_{2}$ for each $n \geq 1$. So, utilizing (24), 
(63)-(66), from $\left\{\lambda_{n}\right\} \subset(0, b] \subset(0,1),\left\{\alpha_{n}\right\} \subset(0,1]$, $\left\{\rho_{n}\right\} \subset(0,2 \alpha]$, and $\mu \in\left(0,2 \beta / L^{2}\right)$, we deduce that

$$
\begin{aligned}
\left\|y_{n+1}-y_{n}\right\| & \\
= & \left\|W_{n+1} G \widetilde{v}_{n+1}-W_{n} G \widetilde{v}_{n}\right\| \\
\leq & \left\|W_{n+1} G \widetilde{v}_{n+1}-W_{n+1} G \widetilde{v}_{n}\right\|+\left\|W_{n+1} G \widetilde{v}_{n}-W_{n} G \widetilde{v}_{n}\right\| \\
\leq & \left\|\widetilde{v}_{n+1}-\widetilde{v}_{n}\right\|+\widetilde{M}_{2} \prod_{i=1}^{n} \lambda_{i} \\
\leq & \left\|\widetilde{v}_{n+1}-\widetilde{v}_{n}\right\|+\widetilde{M}_{2} b^{n} \\
\leq & \widetilde{M}_{0} \sum_{i=1}^{N}\left|\lambda_{i, n+1}-\lambda_{i, n}\right|+\left\|u_{n+1}-u_{n}\right\| \\
& +\left|\rho_{n+1}-\rho_{n}\right|\left\|\widetilde{A}_{1} v_{n}\right\|+\widetilde{M}_{2} b^{n} \\
\leq & \widetilde{M}_{0} \sum_{i=1}^{N}\left|\lambda_{i, n+1}-\lambda_{i, n}\right| \\
& +\widetilde{M}_{1} \sum_{k=1}^{M}\left|r_{k, n+1}-r_{k, n}\right|+\left\|x_{n+1}-x_{n}\right\| \\
& +\left|\rho_{n+1}-\rho_{n}\right|\left\|\widetilde{A}_{1} v_{n}\right\|+\widetilde{M}_{2} b^{n},
\end{aligned}
$$

and, by Lemma 9,

$$
\begin{aligned}
\| x_{n+2} & -x_{n+1} \| \\
= & \left\|\left(I-\mu \alpha_{n+1} \widetilde{A}_{2}\right) W_{n+1} G \widetilde{v}_{n+1}-\left(I-\mu \alpha_{n} \widetilde{A}_{2}\right) W_{n} G \widetilde{v}_{n}\right\| \\
\leq & \left\|\left(I-\mu \alpha_{n+1} \widetilde{A}_{2}\right) W_{n+1} G \widetilde{v}_{n+1}-\left(I-\mu \alpha_{n+1} \widetilde{A}_{2}\right) W_{n} G \widetilde{v}_{n}\right\| \\
& +\left\|\left(I-\mu \alpha_{n+1} \widetilde{A}_{2}\right) W_{n} G \widetilde{v}_{n}-\left(I-\mu \alpha_{n} \widetilde{A}_{2}\right) W_{n} G \widetilde{v}_{n}\right\| \\
\leq & \left(1-\alpha_{n+1} \tau\right)\left\|W_{n+1} G \widetilde{v}_{n+1}-W_{n} G \widetilde{v}_{n}\right\| \\
& +\left|\alpha_{n+1}-\alpha_{n}\right| \mu\left\|\widetilde{A}_{2} W_{n} G \widetilde{v}_{n}\right\| \\
\leq & \left(1-\alpha_{n+1} \tau\right) \\
& \quad\left[\widetilde{M}_{0} \sum_{i=1}^{N}\left|\lambda_{i, n+1}-\lambda_{i, n}\right|\right. \\
& +\widetilde{M}_{1} \sum_{k=1}^{M}\left|r_{k, n+1}-r_{k, n}\right|+\left\|x_{n+1}-x_{n}\right\| \\
& \left.+\left|\rho_{n+1}-\rho_{n}\right|\left\|\widetilde{A}_{1} v_{n}\right\|+\widetilde{M}_{2} b^{n}\right] \\
& +\left|\alpha_{n+1}-\alpha_{n}\right| \mu\left\|\widetilde{A}_{2} W_{n} G \widetilde{v}_{n}\right\| \\
\leq & \left(1-\alpha_{n+1} \tau\right)\left\|x_{n+1}-x_{n}\right\|
\end{aligned}
$$

$$
\begin{aligned}
& +\widetilde{M}_{0} \sum_{i=1}^{N}\left|\lambda_{i, n+1}-\lambda_{i, n}\right|+\widetilde{M}_{1} \sum_{k=1}^{M}\left|r_{k, n+1}-r_{k, n}\right| \\
& +\left|\rho_{n+1}-\rho_{n}\right|\left\|\widetilde{A}_{1} v_{n}\right\|+\widetilde{M}_{2} b^{n} \\
& +\left|\alpha_{n+1}-\alpha_{n}\right| \mu\left\|\widetilde{A}_{2} W_{n} G \widetilde{v}_{n}\right\| \\
& \leq\left(1-\alpha_{n+1} \tau\right)\left\|x_{n+1}-x_{n}\right\| \\
& +\widetilde{M}_{3}\left(\sum_{i=1}^{N}\left|\lambda_{i, n+1}-\lambda_{i, n}\right|+\sum_{k=1}^{M}\left|r_{k, n+1}-r_{k, n}\right|\right. \\
& \left.\quad+\left|\rho_{n+1}-\rho_{n}\right|+\left|\alpha_{n+1}-\alpha_{n}\right|+b^{n}\right),
\end{aligned}
$$

where $\sup _{n \geq 1}\left\{\widetilde{M}_{0}+\widetilde{M}_{1}+\widetilde{M}_{2}+\left\|\widetilde{A}_{1} v_{n}\right\|+\mu\left\|\widetilde{A}_{2} W_{n} G \widetilde{v}_{n}\right\|\right\} \leq \widetilde{M}_{3}$ for some $\widetilde{M}_{3}>0$. Consequently,

$$
\begin{aligned}
& \frac{\left\|x_{n+1}-x_{n}\right\|}{\rho_{n}} \\
& \leq\left(1-\alpha_{n} \tau\right) \frac{\left\|x_{n}-x_{n-1}\right\|}{\rho_{n}} \\
& +\widetilde{M}_{3}\left(\sum_{i=1}^{N} \frac{\left|\lambda_{i, n}-\lambda_{i, n-1}\right|}{\rho_{n}}+\sum_{k=1}^{M} \frac{\left|r_{k, n}-r_{k, n-1}\right|}{\rho_{n}}\right. \\
& \left.+\frac{\left|\rho_{n}-\rho_{n-1}\right|}{\rho_{n}}+\frac{\left|\alpha_{n}-\alpha_{n-1}\right|}{\rho_{n}}+\frac{b^{n-1}}{\rho_{n}}\right) \\
& =\left(1-\tau \alpha_{n}\right) \frac{|| x_{n}-x_{n-1} \mid}{\rho_{n-1}} \\
& +\left(1-\tau \alpha_{n}\right)|| x_{n}-x_{n-1} \mid\left(\frac{1}{\rho_{n}}-\frac{1}{\rho_{n-1}}\right) \\
& +\widetilde{M}_{3}\left(\sum_{i=1}^{N} \frac{\left|\lambda_{i, n}-\lambda_{i, n-1}\right|}{\rho_{n}}+\sum_{k=1}^{M} \frac{\left|r_{k, n}-r_{k, n-1}\right|}{\rho_{n}}\right. \\
& \left.+\frac{\left|\rho_{n}-\rho_{n-1}\right|}{\rho_{n}}+\frac{\left|\alpha_{n}-\alpha_{n-1}\right|}{\rho_{n}}+\frac{b^{n-1}}{\rho_{n}}\right) \\
& +\left(1-\tau \alpha_{n}\right) \frac{\left\|x_{n}-x_{n-1}\right\|}{\rho_{n-1}} \\
& +\tau \alpha_{n} \cdot \frac{\widetilde{M}_{4}}{\tau}\left\{\frac{1}{\alpha_{n}}\left|\frac{1}{\rho_{n}}-\frac{1}{\rho_{n-1}}\right|\right. \\
& +\sum_{i=1}^{N} \frac{\left|\lambda_{i, n}-\lambda_{i, n-1}\right|}{\alpha_{n} \rho_{n}} \\
& +\frac{1}{\rho_{n}}\left|1-\frac{\alpha_{k, n}-r_{k, n-1} \mid}{\alpha_{n} \rho_{n}}+\frac{1}{\alpha_{n}}\right| 1-\frac{\rho_{n-1}}{\rho_{n}} \mid \\
& \left.\alpha_{n} \rho_{n}\right\}
\end{aligned}
$$


where $\sup _{n \geq 1}\left\{\left\|x_{n+1}-x_{n}\right\|+\widetilde{M}_{3}\right\} \leq \widetilde{M}_{4}$ for some $\widetilde{M}_{4}>0$. From (H1)-(H5), it follows that $\sum_{n=1}^{\infty} \tau \alpha_{n}=\infty$ and

$$
\begin{gathered}
\lim _{n \rightarrow \infty} \frac{\widetilde{M}_{4}}{\tau}\left\{\frac{1}{\alpha_{n}}\left|\frac{1}{\rho_{n}}-\frac{1}{\rho_{n-1}}\right|+\sum_{i=1}^{N} \frac{\left|\lambda_{i, n}-\lambda_{i, n-1}\right|}{\alpha_{n} \rho_{n}}\right. \\
+\sum_{k=1}^{M} \frac{\left|r_{k, n}-r_{k, n-1}\right|}{\alpha_{n} \rho_{n}}+\frac{1}{\alpha_{n}}\left|1-\frac{\rho_{n-1}}{\rho_{n}}\right| \\
\left.+\frac{1}{\rho_{n}}\left|1-\frac{\alpha_{n-1}}{\alpha_{n}}\right|+\frac{b^{n-1}}{\alpha_{n} \rho_{n}}\right\}=0 .
\end{gathered}
$$

Thus, applying Lemma 10 to (69), we immediately conclude that

$$
\lim _{n \rightarrow \infty} \frac{\left\|x_{n+1}-x_{n}\right\|}{\rho_{n}}=0
$$

So, from (H3), it follows that

$$
\lim _{n \rightarrow \infty}\left\|x_{n+1}-x_{n}\right\|=0
$$

Step 3. We prove that $\lim _{n \rightarrow \infty}\left\|x_{n}-u_{n}\right\|=0, \lim _{n \rightarrow \infty} \| x_{n}-$ $v_{n}\left\|=0, \lim _{n \rightarrow \infty}\right\| \widetilde{v}_{n}-G \widetilde{v}_{n} \|=0$, and $\lim _{n \rightarrow \infty}\left\|\widetilde{v}_{n}-W \widetilde{v}_{n}\right\|=0$, where $\widetilde{v}_{n}=v_{n}-\rho_{n} \widetilde{A}_{1} v_{n}$.

Indeed, utilizing Lemma 4, from (48), we get

$$
\begin{aligned}
\left\|x_{n+1}-x^{*}\right\|^{2} & \\
= & \left\|\left(I-\mu \alpha_{n} \widetilde{A}_{2}\right) W_{n} G \widetilde{v}_{n}-x^{*}\right\|^{2} \\
= & \|\left(I-\mu \alpha_{n} \widetilde{A}_{2}\right) W_{n} G \widetilde{v}_{n}-\left(I-\mu \alpha_{n} \widetilde{A}_{2}\right) W_{n} G x^{*} \\
& +\left(I-\mu \alpha_{n} \widetilde{A}_{2}\right) W_{n} G x^{*}-W_{n} G x^{*} \|^{2} \\
\leq & \left\|\left(I-\mu \alpha_{n} \widetilde{A}_{2}\right) W_{n} G \widetilde{v}_{n}-\left(I-\mu \alpha_{n} \widetilde{A}_{2}\right) W_{n} G x^{*}\right\|^{2} \\
& -2 \mu \alpha_{n}\left\langle\widetilde{A}_{2} x^{*}, x_{n+1}-x^{*}\right\rangle \\
\leq & \left(1-\alpha_{n} \tau\right)\left\|G \widetilde{v}_{n}-x^{*}\right\|^{2}-2 \mu \alpha_{n}\left\langle\widetilde{A}_{2} x^{*}, x_{n+1}-x^{*}\right\rangle \\
\leq & \left(1-\alpha_{n} \tau\right)\left\|\widetilde{v}_{n}-x^{*}\right\|^{2}-2 \mu \alpha_{n}\left\langle\widetilde{A}_{2} x^{*}, x_{n+1}-x^{*}\right\rangle \\
= & \left(1-\alpha_{n} \tau\right)\left\|v_{n}-x^{*}-\rho_{n} \widetilde{A}_{1} v_{n}\right\|^{2} \\
& -2 \mu \alpha_{n}\left\langle\widetilde{A}_{2} x^{*}, x_{n+1}-x^{*}\right\rangle \\
\leq & \left(1-\alpha_{n} \tau\right)\left(\left\|v_{n}-x^{*}\right\|^{2}-2 \rho_{n}\left\langle\widetilde{A}_{1} v_{n}, \widetilde{v}_{n}-x^{*}\right\rangle\right) \\
& -2 \mu \alpha_{n}\left\langle\widetilde{A}_{2} x^{*}, x_{n+1}-x^{*}\right\rangle \\
\leq & \left\|v_{n}-x^{*}\right\|^{2}-2\left(1-\alpha_{n} \tau\right) \rho_{n}\left\langle\widetilde{A}_{1} v_{n}, \widetilde{v}_{n}-x^{*}\right\rangle \\
& -2 \mu \alpha_{n}\left\langle\widetilde{A}_{2} x^{*}, x_{n+1}-x^{*}\right\rangle .
\end{aligned}
$$

On the other hand, observe that

$$
\begin{aligned}
& \left\|\Delta_{n}^{k} x_{n}-x^{*}\right\|^{2} \\
& \quad=\left\|T_{\left.r_{k, n}^{(\Theta}, \varphi_{k}\right)}^{\left(\Theta^{\prime}\right.}\left(I-r_{k, n} A_{k}\right) \Delta_{n}^{k-1} x_{n}-T_{r_{k, n}}^{\left(\Theta_{k}, \varphi_{k}\right)}\left(I-r_{k, n} A_{k}\right) x^{*}\right\|^{2} \\
& \quad \leq\left\|\left(I-r_{k, n} A_{k}\right) \Delta_{n}^{k-1} x_{n}-\left(I-r_{k, n} A_{k}\right) x^{*}\right\|^{2} \\
& \quad \leq\left\|\Delta_{n}^{k-1} x_{n}-x^{*}\right\|^{2}+r_{k, n}\left(r_{k, n}-2 \mu_{k}\right)\left\|A_{k} \Delta_{n}^{k-1} x_{n}-A_{k} x^{*}\right\|^{2} \\
& \quad \leq\left\|x_{n}-x^{*}\right\|^{2}+r_{k, n}\left(r_{k, n}-2 \mu_{k}\right)\left\|A_{k} \Delta_{n}^{k-1} x_{n}-A_{k} x^{*}\right\|^{2},
\end{aligned}
$$

$$
\begin{aligned}
& \left\|\Lambda_{n}^{i} u_{n}-x^{*}\right\|^{2} \\
& \quad=\left\|J_{R_{i}, \lambda_{i, n}}\left(I-\lambda_{i, n} B_{i}\right) \Lambda_{n}^{i-1} u_{n}-J_{R_{i}, \lambda_{i, n}}\left(I-\lambda_{i, n} B_{i}\right) x^{*}\right\|^{2} \\
& \quad \leq\left\|\left(I-\lambda_{i, n} B_{i}\right) \Lambda_{n}^{i-1} u_{n}-\left(I-\lambda_{i, n} B_{i}\right) x^{*}\right\|^{2} \\
& \quad \leq\left\|\Lambda_{n}^{i-1} u_{n}-x^{*}\right\|^{2}+\lambda_{i, n}\left(\lambda_{i, n}-2 \eta_{i}\right)\left\|B_{i} \Lambda_{n}^{i-1} u_{n}-B_{i} x^{*}\right\|^{2} \\
& \quad \leq\left\|u_{n}-x^{*}\right\|^{2}+\lambda_{i, n}\left(\lambda_{i, n}-2 \eta_{i}\right)\left\|B_{i} \Lambda_{n}^{i-1} u_{n}-B_{i} x^{*}\right\|^{2} \\
& \quad \leq\left\|x_{n}-x^{*}\right\|^{2}+\lambda_{i, n}\left(\lambda_{i, n}-2 \eta_{i}\right)\left\|B_{i} \Lambda_{n}^{i-1} u_{n}-B_{i} x^{*}\right\|^{2} .
\end{aligned}
$$

Combining (73)-(75), we get

$$
\begin{aligned}
\left\|x_{n+1}-x^{*}\right\|^{2} & \\
\leq & \left\|v_{n}-x^{*}\right\|^{2}-2\left(1-\alpha_{n} \tau\right) \rho_{n}\left\langle\widetilde{A}_{1} v_{n}, \widetilde{v}_{n}-x^{*}\right\rangle \\
& -2 \mu \alpha_{n}\left\langle\widetilde{A}_{2} x^{*}, x_{n+1}-x^{*}\right\rangle \\
\leq & \left\|\Lambda_{n}^{i} u_{n}-x^{*}\right\|^{2}+2 \rho_{n}\left\|\widetilde{A}_{1} v_{n}\right\|\left\|\widetilde{v}_{n}-x^{*}\right\| \\
& +2 \mu \alpha_{n}\left\|\widetilde{A}_{2} x^{*}\right\|\left\|x_{n+1}-x^{*}\right\| \\
\leq & \left\|u_{n}-x^{*}\right\|^{2}+\lambda_{i, n}\left(\lambda_{i, n}-2 \eta_{i}\right)\left\|B_{i} \Lambda_{n}^{i-1} u_{n}-B_{i} x^{*}\right\|^{2} \\
& +2 \rho_{n}\left\|\widetilde{A}_{1} v_{n}\right\|\left\|\widetilde{v}_{n}-x^{*}\right\| \\
& +2 \mu \alpha_{n}\left\|\widetilde{A}_{2} x^{*}\right\|\left\|x_{n+1}-x^{*}\right\| \\
\leq & \left\|\Delta_{n}^{k} x_{n}-x^{*}\right\|^{2}+\lambda_{i, n}\left(\lambda_{i, n}-2 \eta_{i}\right)\left\|B_{i} \Lambda_{n}^{i-1} u_{n}-B_{i} x^{*}\right\|^{2} \\
& +2 \rho_{n}\left\|\widetilde{A}_{1} v_{n}\right\|\left\|\widetilde{v}_{n}-x^{*}\right\| \\
& +2 \mu \alpha_{n}\left\|\widetilde{A}_{2} x^{*}\right\|\left\|x_{n+1}-x^{*}\right\| \\
\leq & \left\|x_{n}-x^{*}\right\|^{2}+r_{k, n}\left(r_{k, n}-2 \mu_{k}\right)\left\|A_{k} \Delta_{n}^{k-1} x_{n}-A_{k} x^{*}\right\|^{2} \\
& +\lambda_{i, n}\left(\lambda_{i, n}-2 \eta_{i}\right)\left\|B_{i} \Lambda_{n}^{i-1} u_{n}-B_{i} x^{*}\right\|^{2} \\
& +2 \rho_{n}\left\|\widetilde{A}_{1} v_{n}\right\|\left\|\widetilde{v}_{n}-x^{*}\right\|+2 \mu \alpha_{n}\left\|\widetilde{A}_{2} x^{*}\right\|\left\|x_{n+1}-x^{*}\right\|,
\end{aligned}
$$


Journal of Function Spaces

13

which leads to

$$
\begin{aligned}
& r_{k, n}\left(2 \mu_{k}-r_{k, n}\right)\left\|A_{k} \Delta_{n}^{k-1} x_{n}-A_{k} x^{*}\right\|^{2} \\
& \quad+\lambda_{i, n}\left(2 \eta_{i}-\lambda_{i, n}\right)\left\|B_{i} \Lambda_{n}^{i-1} u_{n}-B_{i} x^{*}\right\|^{2} \\
& \leq\left\|x_{n}-x^{*}\right\|^{2}-\left\|x_{n+1}-x^{*}\right\|^{2}+2 \rho_{n}\left\|\widetilde{A}_{1} v_{n}\right\|\left\|\widetilde{v}_{n}-x^{*}\right\| \\
& \quad+2 \mu \alpha_{n}\left\|\widetilde{A}_{2} x^{*}\right\|\left\|x_{n+1}-x^{*}\right\| \\
& \leq\left\|x_{n}-x_{n+1}\right\|\left(\left\|x_{n}-x^{*}\right\|+\left\|x_{n+1}-x^{*}\right\|\right) \\
& \quad+2 \rho_{n}\left\|\widetilde{A}_{1} v_{n}\right\|\left\|\widetilde{v}_{n}-x^{*}\right\|+2 \mu \alpha_{n}\left\|\widetilde{A}_{2} x^{*}\right\|\left\|x_{n+1}-x^{*}\right\| .
\end{aligned}
$$

Since $\alpha_{n} \rightarrow 0, \rho_{n} \rightarrow 0,\left\|x_{n+1}-x_{n}\right\| \rightarrow 0$, and $\left\{x_{n}\right\},\left\{v_{n}\right\},\{\widetilde{v}\}$ are bounded sequences, it follows from $\left\{\lambda_{i, n}\right\} \subset\left[a_{i}, b_{i}\right] \subset$ $\left(0,2 \eta_{i}\right)$ and $\left\{r_{k, n}\right\} \subset\left[e_{k}, f_{k}\right] \subset\left(0,2 \mu_{k}\right)$ that

$$
\begin{aligned}
& \lim _{n \rightarrow \infty}\left\|A_{k} \Delta_{n}^{k-1} x_{n}-A_{k} x^{*}\right\|=0, \\
& \lim _{n \rightarrow \infty}\left\|B_{i} \Lambda_{n}^{i-1} u_{n}-B_{i} x^{*}\right\|=0,
\end{aligned}
$$

for all $k \in\{1,2, \ldots, M\}$ and $i \in\{1,2, \ldots, N\}$.

Furthermore, by Proposition 3(ii) and Lemma 11 (a), we have

$$
\begin{aligned}
& \left\|\Delta_{n}^{k} x_{n}-x^{*}\right\|^{2} \\
& =\left\|T_{r_{k, n}}^{\left(\Theta_{k}, \varphi_{k}\right)}\left(I-r_{k, n} A_{k}\right) \Delta_{n}^{k-1} x_{n}-T_{r_{k, n}}^{\left(\Theta_{k}, \varphi_{k}\right)}\left(I-r_{k, n} A_{k}\right) x^{*}\right\|^{2} \\
& \leq\left\langle\left(I-r_{k, n} A_{k}\right) \Delta_{n}^{k-1} x_{n}-\left(I-r_{k, n} A_{k}\right) x^{*}, \Delta_{n}^{k} x_{n}-x^{*}\right\rangle \\
& =\frac{1}{2}\left(\left\|\left(I-r_{k, n} A_{k}\right) \Delta_{n}^{k-1} x_{n}-\left(I-r_{k, n} A_{k}\right) x^{*}\right\|^{2}\right. \\
& \quad+\left\|\Delta_{n}^{k} x_{n}-x^{*}\right\|^{2} \\
& \quad-\|\left(I-r_{k, n} A_{k}\right) \Delta_{n}^{k-1} x_{n}-\left(I-r_{k, n} A_{k}\right) x^{*} \\
& \left.\quad-\left(\Delta_{n}^{k} x_{n}-x^{*}\right) \|^{2}\right) \\
& \left.\quad-\left\|\Delta_{n}^{k-1} x_{n}-\Delta_{n}^{k} x_{n}-r_{k, n}\left(A_{k} \Delta_{n}^{k-1} x_{n}-A_{k} x^{*}\right)\right\|^{2}\right) \\
& \leq \frac{1}{2}\left(\left\|\Delta_{n}^{k-1} x_{n}-x^{*}\right\|^{2}+\left\|\Delta_{n}^{k} x_{n}-x^{*}\right\|^{2}\right.
\end{aligned}
$$

which implies that

$$
\begin{aligned}
\left\|\Delta_{n}^{k} x_{n}-x^{*}\right\|^{2} & \\
\leq & \left\|\Delta_{n}^{k-1} x_{n}-x^{*}\right\|^{2} \\
& -\left\|\Delta_{n}^{k-1} x_{n}-\Delta_{n}^{k} x_{n}-r_{k, n}\left(A_{k} \Delta_{n}^{k-1} x_{n}-A_{k} x^{*}\right)\right\|^{2} \\
= & \left\|\Delta_{n}^{k-1} x_{n}-x^{*}\right\|^{2}-\left\|\Delta_{n}^{k-1} x_{n}-\Delta_{n}^{k} x_{n}\right\|^{2} \\
& -r_{k, n}^{2}\left\|A_{k} \Delta_{n}^{k-1} x_{n}-A_{k} x^{*}\right\|^{2} \\
& +2 r_{k, n}\left\langle\Delta_{n}^{k-1} x_{n}-\Delta_{n}^{k} x_{n}, A_{k} \Delta_{n}^{k-1} x_{n}-A_{k} x^{*}\right\rangle \\
\leq & \left\|\Delta_{n}^{k-1} x_{n}-x^{*}\right\|^{2}-\left\|\Delta_{n}^{k-1} x_{n}-\Delta_{n}^{k} x_{n}\right\|^{2} \\
& +2 r_{k, n}\left\|\Delta_{n}^{k-1} x_{n}-\Delta_{n}^{k} x_{n}\right\|\left\|A_{k} \Delta_{n}^{k-1} x_{n}-A_{k} x^{*}\right\| \\
\leq & \left\|x_{n}-x^{*}\right\|^{2}-\left\|\Delta_{n}^{k-1} x_{n}-\Delta_{n}^{k} x_{n}\right\|^{2} \\
& +2 r_{k, n}\left\|\Delta_{n}^{k-1} x_{n}-\Delta_{n}^{k} x_{n}\right\|\left\|A_{k} \Delta_{n}^{k-1} x_{n}-A_{k} x^{*}\right\| .
\end{aligned}
$$

By Lemma 11 (a) and Lemma 14, we obtain

$$
\begin{aligned}
& \left\|\Lambda_{n}^{i} u_{n}-x^{*}\right\|^{2} \\
& =\left\|J_{R_{i}, \lambda_{i, n}}\left(I-\lambda_{i, n} B_{i}\right) \Lambda_{n}^{i-1} u_{n}-J_{R_{i}, \lambda_{i, n}}\left(I-\lambda_{i, n} B_{i}\right) x^{*}\right\|^{2} \\
& \leq\left\langle\left(I-\lambda_{i, n} B_{i}\right) \Lambda_{n}^{i-1} u_{n}-\left(I-\lambda_{i, n} B_{i}\right) x^{*}, \Lambda_{n}^{i} u_{n}-x^{*}\right\rangle \\
& =\frac{1}{2}\left(\left\|\left(I-\lambda_{i, n} B_{i}\right) \Lambda_{n}^{i-1} u_{n}-\left(I-\lambda_{i, n} B_{i}\right) x^{*}\right\|^{2}\right. \\
& +\left\|\Lambda_{n}^{i} u_{n}-x^{*}\right\|^{2} \\
& -\|\left(I-\lambda_{i, n} B_{i}\right) \Lambda_{n}^{i-1} u_{n} \\
& \left.-\left(I-\lambda_{i, n} B_{i}\right) x^{*}-\left(\Lambda_{n}^{i} u_{n}-x^{*}\right) \|^{2}\right) \\
& \leq \frac{1}{2}\left(\left\|\Lambda_{n}^{i-1} u_{n}-x^{*}\right\|^{2}+\left\|\Lambda_{n}^{i} u_{n}-x^{*}\right\|^{2}\right. \\
& \left.-\left\|\Lambda_{n}^{i-1} u_{n}-\Lambda_{n}^{i} u_{n}-\lambda_{i, n}\left(B_{i} \Lambda_{n}^{i-1} u_{n}-B_{i} x^{*}\right)\right\|^{2}\right) \\
& \leq \frac{1}{2}\left(\left\|u_{n}-x^{*}\right\|^{2}+\left\|\Lambda_{n}^{i} u_{n}-x^{*}\right\|^{2}\right. \\
& \left.-\left\|\Lambda_{n}^{i-1} u_{n}-\Lambda_{n}^{i} u_{n}-\lambda_{i, n}\left(B_{i} \Lambda_{n}^{i-1} u_{n}-B_{i} x^{*}\right)\right\|^{2}\right) \\
& \leq \frac{1}{2}\left(\left\|x_{n}-x^{*}\right\|^{2}+\left\|\Lambda_{n}^{i} u_{n}-x^{*}\right\|^{2}\right. \\
& \left.-\left\|\Lambda_{n}^{i-1} u_{n}-\Lambda_{n}^{i} u_{n}-\lambda_{i, n}\left(B_{i} \Lambda_{n}^{i-1} u_{n}-B_{i} x^{*}\right)\right\|^{2}\right),
\end{aligned}
$$


which implies

$$
\begin{aligned}
\left\|\Lambda_{n}^{i} u_{n}-x^{*}\right\|^{2} \\
\leq\left\|x_{n}-x^{*}\right\|^{2} \\
\quad-\left\|\Lambda_{n}^{i-1} u_{n}-\Lambda_{n}^{i} u_{n}-\lambda_{i, n}\left(B_{i} \Lambda_{n}^{i-1} u_{n}-B_{i} x^{*}\right)\right\|^{2} \\
=\left\|x_{n}-x^{*}\right\|^{2}-\left\|\Lambda_{n}^{i-1} u_{n}-\Lambda_{n}^{i} u_{n}\right\|^{2} \\
\quad-\lambda_{i, n}^{2}\left\|B_{i} \Lambda_{n}^{i-1} u_{n}-B_{i} x^{*}\right\|^{2} \\
\quad+2 \lambda_{i, n}\left\langle\Lambda_{n}^{i-1} u_{n}-\Lambda_{n}^{i} u_{n}, B_{i} \Lambda_{n}^{i-1} u_{n}-B_{i} x^{*}\right\rangle \\
\leq\left\|x_{n}-x^{*}\right\|^{2}-\left\|\Lambda_{n}^{i-1} u_{n}-\Lambda_{n}^{i} u_{n}\right\|^{2} \\
\quad+2 \lambda_{i, n}\left\|\Lambda_{n}^{i-1} u_{n}-\Lambda_{n}^{i} u_{n}\right\|\left\|B_{i} \Lambda_{n}^{i-1} u_{n}-B_{i} x^{*}\right\| .
\end{aligned}
$$

Combining (73) and (82), we conclude that

$$
\begin{aligned}
& \left\|x_{n+1}-x^{*}\right\|^{2} \\
& \leq\left\|v_{n}-x^{*}\right\|^{2}-2\left(1-\alpha_{n} \tau\right) \rho_{n}\left\langle\widetilde{A}_{1} v_{n}, \widetilde{v}_{n}-x^{*}\right\rangle \\
& \quad-2 \mu \alpha_{n}\left\langle\widetilde{A}_{2} x^{*}, x_{n+1}-x^{*}\right\rangle \\
& \leq\left\|\Lambda_{n}^{i} u_{n}-x^{*}\right\|^{2}+2 \rho_{n}\left\|\widetilde{A}_{1} v_{n}\right\|\left\|\widetilde{v}_{n}-x^{*}\right\| \\
& \quad+2 \mu \alpha_{n}\left\|\widetilde{A}_{2} x^{*}\right\|\left\|x_{n+1}-x^{*}\right\| \\
& \leq\left\|x_{n}-x^{*}\right\|^{2}-\left\|\Lambda_{n}^{i-1} u_{n}-\Lambda_{n}^{i} u_{n}\right\|^{2} \\
& \quad+2 \lambda_{i, n}\left\|\Lambda_{n}^{i-1} u_{n}-\Lambda_{n}^{i} u_{n}\right\|\left\|B_{i} \Lambda_{n}^{i-1} u_{n}-B_{i} x^{*}\right\| \\
& \quad+2 \rho_{n}\left\|\widetilde{A}_{1} v_{n}\right\|\left\|\widetilde{v}_{n}-x^{*}\right\|+2 \mu \alpha_{n}\left\|\widetilde{A}_{2} x^{*}\right\|\left\|x_{n+1}-x^{*}\right\|
\end{aligned}
$$

which yields

$$
\begin{aligned}
&\left\|\Lambda_{n}^{i-1} u_{n}-\Lambda_{n}^{i} u_{n}\right\|^{2} \\
& \leq\left\|x_{n}-x^{*}\right\|^{2}-\left\|x_{n+1}-x^{*}\right\|^{2} \\
& \quad+2 \lambda_{i, n}\left\|\Lambda_{n}^{i-1} u_{n}-\Lambda_{n}^{i} u_{n}\right\|\left\|B_{i} \Lambda_{n}^{i-1} u_{n}-B_{i} x^{*}\right\| \\
& \quad+2 \rho_{n}\left\|\widetilde{A}_{1} v_{n}\right\|\left\|\widetilde{v}_{n}-x^{*}\right\|+2 \mu \alpha_{n}\left\|\widetilde{A}_{2} x^{*}\right\|\left\|x_{n+1}-x^{*}\right\| \\
& \leq\left\|x_{n}-x_{n+1}\right\|\left(\left\|x_{n}-x^{*}\right\|+\left\|x_{n+1}-x^{*}\right\|\right) \\
&+2 \lambda_{i, n}\left\|\Lambda_{n}^{i-1} u_{n}-\Lambda_{n}^{i} u_{n}\right\|\left\|B_{i} \Lambda_{n}^{i-1} u_{n}-B_{i} x^{*}\right\| \\
&+2 \rho_{n}\left\|\widetilde{A}_{1} v_{n}\right\|\left\|\widetilde{v}_{n}-x^{*}\right\|+2 \mu \alpha_{n}\left\|\widetilde{A}_{2} x^{*}\right\|\left\|x_{n+1}-x^{*}\right\| .
\end{aligned}
$$

Since $\alpha_{n} \rightarrow 0, \rho_{n} \rightarrow 0$, and $\left\|x_{n+1}-x_{n}\right\| \rightarrow 0$ and $\left\{x_{n}\right\}$, $\left\{v_{n}\right\}$, and $\{\widetilde{v}\}$ are bounded sequences, it follows from (78) and $\left\{\lambda_{i, n}\right\} \subset\left[a_{i}, b_{i}\right] \subset\left(0,2 \eta_{i}\right)$ that

$$
\lim _{n \rightarrow \infty}\left\|\Lambda_{n}^{i-1} u_{n}-\Lambda_{n}^{i} u_{n}\right\|=0, \quad \forall i \in\{1,2, \ldots, N\} .
$$

Also, combining (55), (73), and (80), we deduce that

$$
\begin{aligned}
\left\|x_{n+1}-x^{*}\right\|^{2} & \left\|v_{n}-x^{*}\right\|^{2}-2\left(1-\alpha_{n} \tau\right) \rho_{n}\left\langle\widetilde{A}_{1} v_{n}, \widetilde{v}_{n}-x^{*}\right\rangle \\
& -2 \mu \alpha_{n}\left\langle\widetilde{A}_{2} x^{*}, x_{n+1}-x^{*}\right\rangle \\
\leq & \left\|u_{n}-x^{*}\right\|^{2}+2 \rho_{n}\left\|\widetilde{A}_{1} v_{n}\right\|\left\|\widetilde{v}_{n}-x^{*}\right\| \\
& +2 \mu \alpha_{n}\left\|\widetilde{A}_{2} x^{*}\right\|\left\|x_{n+1}-x^{*}\right\| \\
\leq & \left\|\Delta_{n}^{k} x_{n}-x^{*}\right\|^{2}+2 \rho_{n}\left\|\widetilde{A}_{1} v_{n}\right\|\left\|\widetilde{v}_{n}-x^{*}\right\| \\
& +2 \mu \alpha_{n}\left\|\widetilde{A}_{2} x^{*}\right\|\left\|x_{n+1}-x^{*}\right\| \\
\leq & \left\|x_{n}-x^{*}\right\|^{2}-\left\|\Delta_{n}^{k-1} x_{n}-\Delta_{n}^{k} x_{n}\right\|^{2} \\
& +2 r_{k, n}\left\|\Delta_{n}^{k-1} x_{n}-\Delta_{n}^{k} x_{n}\right\|\left\|A_{k} \Delta_{n}^{k-1} x_{n}-A_{k} x^{*}\right\| \\
& +2 \rho_{n}\left\|\widetilde{A}_{1} v_{n}\right\|\left\|\widetilde{v}_{n}-x^{*}\right\|+2 \mu \alpha_{n}\left\|\widetilde{A}_{2} x^{*}\right\|\left\|x_{n+1}-x^{*}\right\|,
\end{aligned}
$$

which leads to

$$
\begin{aligned}
& \left\|\Delta_{n}^{k-1} x_{n}-\Delta_{n}^{k} x_{n}\right\|^{2} \\
& \leq\left\|x_{n}-x^{*}\right\|^{2}-\left\|x_{n+1}-x^{*}\right\|^{2} \\
& \quad+2 r_{k, n}\left\|\Delta_{n}^{k-1} x_{n}-\Delta_{n}^{k} x_{n}\right\|\left\|A_{k} \Delta_{n}^{k-1} x_{n}-A_{k} x^{*}\right\| \\
& \quad+2 \rho_{n}\left\|\widetilde{A}_{1} v_{n}\right\|\left\|\widetilde{v}_{n}-x^{*}\right\|+2 \mu \alpha_{n}\left\|\widetilde{A}_{2} x^{*}\right\|\left\|x_{n+1}-x^{*}\right\| \\
& \leq\left\|x_{n}-x_{n+1}\right\|\left(\left\|x_{n}-x^{*}\right\|+\left\|x_{n+1}-x^{*}\right\|\right) \\
& \quad+2 r_{k, n}\left\|\Delta_{n}^{k-1} x_{n}-\Delta_{n}^{k} x_{n}\right\|\left\|A_{k} \Delta_{n}^{k-1} x_{n}-A_{k} x^{*}\right\| \\
& \quad+2 \rho_{n}\left\|\widetilde{A}_{1} v_{n}\right\|\left\|\widetilde{v}_{n}-x^{*}\right\|+2 \mu \alpha_{n}\left\|\widetilde{A}_{2} x^{*}\right\|\left\|x_{n+1}-x^{*}\right\| .
\end{aligned}
$$

Since $\alpha_{n} \rightarrow 0, \rho_{n} \rightarrow 0$, and $\left\|x_{n+1}-x_{n}\right\| \rightarrow 0$ and $\left\{x_{n}\right\}$, $\left\{v_{n}\right\}$ and, $\{\widetilde{v}\}$ are bounded sequences, it follows from (78) and $\left\{r_{k, n}\right\} \subset\left[e_{k}, f_{k}\right] \subset\left(0,2 \mu_{k}\right)$ that

$$
\lim _{n \rightarrow \infty}\left\|\Delta_{n}^{k-1} x_{n}-\Delta_{n}^{k} x_{n}\right\|=0, \quad \forall k \in\{1,2, \ldots, M\}
$$

Hence, from (85) and (88), we get

$$
\begin{aligned}
\left\|x_{n}-u_{n}\right\|= & \left\|\Delta_{n}^{0} x_{n}-\Delta_{n}^{M} x_{n}\right\| \\
\leq & \left\|\Delta_{n}^{0} x_{n}-\Delta_{n}^{1} x_{n}\right\|+\left\|\Delta_{n}^{1} x_{n}-\Delta_{n}^{2} x_{n}\right\| \\
& +\cdots+\left\|\Delta_{n}^{M-1} x_{n}-\Delta_{n}^{M} x_{n}\right\| \\
& \longrightarrow 0 \text { as } n \longrightarrow \infty,
\end{aligned}
$$




$$
\begin{aligned}
\left\|u_{n}-v_{n}\right\|= & \left\|\Lambda_{n}^{0} u_{n}-\Lambda_{n}^{N} u_{n}\right\| \\
\leq & \left\|\Lambda_{n}^{0} u_{n}-\Lambda_{n}^{1} u_{n}\right\|+\left\|\Lambda_{n}^{1} u_{n}-\Lambda_{n}^{2} u_{n}\right\| \\
& +\cdots+\left\|\Lambda_{n}^{N-1} u_{n}-\Lambda_{n}^{N} u_{n}\right\| \\
\longrightarrow & 0 \text { as } n \longrightarrow \infty
\end{aligned}
$$

respectively. Thus, from (89) and (90), we obtain

$$
\begin{aligned}
\left\|x_{n}-v_{n}\right\| & \leq\left\|x_{n}-u_{n}\right\|+\left\|u_{n}-v_{n}\right\| \\
& \longrightarrow 0 \quad \text { as } n \longrightarrow \infty .
\end{aligned}
$$

Next, for simplicity, we write $y^{*}=P_{C}\left(I-v_{2} F_{2}\right) x^{*}, w_{n}=$ $P_{C}\left(I-v_{2} F_{2}\right) \widetilde{v}_{n}$, and $\widetilde{w}_{n}=G \widetilde{v}_{n}=P_{C}\left(I-v_{1} F_{1}\right) w_{n}$ for all $n \geq 1$. Then,

$$
\begin{aligned}
x^{*} & =G x^{*}=P_{C}\left(I-v_{1} F_{1}\right) y^{*} \\
& =P_{C}\left(I-v_{1} F_{1}\right) P_{C}\left(I-v_{2} F_{2}\right) x^{*} .
\end{aligned}
$$

We now show that $\lim _{n \rightarrow \infty}\left\|G \widetilde{v}_{n}-\widetilde{v}_{n}\right\|=0$; that is, $\lim _{n \rightarrow \infty}\left\|\widetilde{w}_{n}-\widetilde{v}_{n}\right\|=0$. As a matter of fact, from (56), (57), and (73) it follows that

$$
\begin{aligned}
\| x_{n+1}- & x^{*} \|^{2} \\
\leq & \left(1-\alpha_{n} \tau\right)\left\|G \widetilde{v}_{n}-x^{*}\right\|^{2}-2 \mu \alpha_{n}\left\langle\widetilde{A}_{2} x^{*}, x_{n+1}-x^{*}\right\rangle \\
\leq & \left\|\widetilde{w}_{n}-x^{*}\right\|^{2}+2 \mu \alpha_{n}\left\|\widetilde{A}_{2} x^{*}\right\|\left\|x_{n+1}-x^{*}\right\| \\
\leq & \left\|w_{n}-y^{*}\right\|^{2}+v_{1}\left(v_{1}-2 \zeta_{1}\right)\left\|F_{1} w_{n}-F_{1} y^{*}\right\|^{2} \\
& +2 \mu \alpha_{n}\left\|\widetilde{A}_{2} x^{*}\right\|\left\|x_{n+1}-x^{*}\right\| \\
\leq & \left\|\widetilde{v}_{n}-x^{*}\right\|^{2}+v_{2}\left(v_{2}-2 \zeta_{2}\right)\left\|F_{2} \widetilde{v}_{n}-F_{2} x^{*}\right\|^{2} \\
& +v_{1}\left(v_{1}-2 \zeta_{1}\right)\left\|F_{1} w_{n}-F_{1} y^{*}\right\|^{2} \\
& +2 \mu \alpha_{n}\left\|\widetilde{A}_{2} x^{*}\right\|\left\|x_{n+1}-x^{*}\right\| \\
= & \left\|v_{n}-x^{*}-\rho_{n} \widetilde{A}_{1} v_{n}\right\|^{2} \\
& +v_{2}\left(v_{2}-2 \zeta_{2}\right)\left\|F_{2} \widetilde{v}_{n}-F_{2} x^{*}\right\|^{2} \\
& +v_{1}\left(v_{1}-2 \zeta_{1}\right)\left\|F_{1} w_{n}-F_{1} y^{*}\right\|^{2} \\
& +2 \mu \alpha_{n}\left\|\widetilde{A}_{2} x^{*}\right\|\left\|x_{n+1}-x^{*}\right\| \\
\leq & \left\|v_{n}-x^{*}\right\|^{2}+2 \rho_{n}\left\|\widetilde{A}_{1} v_{n}\right\|\left\|\widetilde{v}_{n}-x^{*}\right\| \\
& +v_{2}\left(v_{2}-2 \zeta_{2}\right)\left\|F_{2} \widetilde{v}_{n}-F_{2} x^{*}\right\|^{2} \\
& +v_{1}\left(v_{1}-2 \zeta_{1}\right)\left\|F_{1} w_{n}-F_{1} y^{*}\right\|^{2} \\
& +2 \mu \alpha_{n}\left\|\widetilde{A}_{2} x^{*}\right\|\left\|x_{n+1}-x^{*}\right\|
\end{aligned}
$$

$$
\begin{aligned}
\leq & \left\|x_{n}-x^{*}\right\|^{2}+2 \rho_{n}\left\|\widetilde{A}_{1} v_{n}\right\|\left\|\widetilde{v}_{n}-x^{*}\right\| \\
& +v_{2}\left(v_{2}-2 \zeta_{2}\right)\left\|F_{2} \widetilde{v}_{n}-F_{2} x^{*}\right\|^{2} \\
& +v_{1}\left(v_{1}-2 \zeta_{1}\right)\left\|F_{1} w_{n}-F_{1} y^{*}\right\|^{2} \\
& +2 \mu \alpha_{n}\left\|\widetilde{A}_{2} x^{*}\right\|\left\|x_{n+1}-x^{*}\right\|,
\end{aligned}
$$

which immediately yields

$$
\begin{aligned}
& v_{2}\left(2 \zeta_{2}-v_{2}\right)\left\|F_{2} \widetilde{v}_{n}-F_{2} x^{*}\right\|^{2}+v_{1}\left(2 \zeta_{1}-v_{1}\right)\left\|F_{1} w_{n}-F_{1} y^{*}\right\|^{2} \\
& \leq\left\|x_{n}-x^{*}\right\|^{2}-\left\|x_{n+1}-x^{*}\right\|^{2}+2 \rho_{n}\left\|\widetilde{A}_{1} v_{n}\right\|\left\|\widetilde{v}_{n}-x^{*}\right\| \\
& \quad+2 \mu \alpha_{n}\left\|\widetilde{A}_{2} x^{*}\right\|\left\|x_{n+1}-x^{*}\right\| \\
& \leq\left\|x_{n}-x_{n+1}\right\|\left(\left\|x_{n}-x^{*}\right\|+\left\|x_{n+1}-x^{*}\right\|\right) \\
& \quad+2 \rho_{n}\left\|\widetilde{A}_{1} v_{n}\right\|\left\|\widetilde{v}_{n}-x^{*}\right\|+2 \mu \alpha_{n}\left\|\widetilde{A}_{2} x^{*}\right\|\left\|x_{n+1}-x^{*}\right\| .
\end{aligned}
$$

Since $\alpha_{n} \rightarrow 0, \rho_{n} \rightarrow 0$, and $\left\|x_{n+1}-x_{n}\right\| \rightarrow 0$ and $\left\{x_{n}\right\},\left\{v_{n}\right\}$, and $\left\{\widetilde{v}_{n}\right\}$ are bounded sequences, it follows from $v_{j} \in\left(0,2 \zeta_{j}\right)$, $j=1,2$, that

$$
\lim _{n \rightarrow \infty}\left\|F_{2} \widetilde{v}_{n}-F_{2} x^{*}\right\|=0, \quad \lim _{n \rightarrow \infty}\left\|F_{1} w_{n}-F_{1} y^{*}\right\|=0 .
$$

In the meantime, in terms of the firm nonexpansivity of $P_{C}$ and the $\zeta_{j}$-inverse strong monotonicity of $F_{j}$ for $j=1,2$, we obtain from $v_{j} \in\left(0,2 \zeta_{j}\right), j=1,2$, and (57) that

$$
\begin{aligned}
& \left\|w_{n}-y^{*}\right\|^{2} \\
& =\left\|P_{C}\left(I-v_{2} F_{2}\right) \widetilde{v}_{n}-P_{C}\left(I-v_{2} F_{2}\right) x^{*}\right\|^{2} \\
& \leq\left\langle\left(I-v_{2} F_{2}\right) \widetilde{v}_{n}-\left(I-v_{2} F_{2}\right) x^{*}, w_{n}-y^{*}\right\rangle \\
& =\frac{1}{2}\left[\left\|\left(I-v_{2} F_{2}\right) \widetilde{v}_{n}-\left(I-v_{2} F_{2}\right) x^{*}\right\|^{2}+\left\|w_{n}-y^{*}\right\|^{2}\right. \\
& \left.\quad-\left\|\left(I-v_{2} F_{2}\right) \widetilde{v}_{n}-\left(I-v_{2} F_{2}\right) x^{*}-\left(w_{n}-y^{*}\right)\right\|^{2}\right] \\
& \leq \frac{1}{2}\left[\left\|\widetilde{v}_{n}-x^{*}\right\|^{2}+\left\|w_{n}-y^{*}\right\|^{2}\right. \\
& \left.\quad-\left\|\left(\widetilde{v}_{n}-w_{n}\right)-v_{2}\left(F_{2} \widetilde{v}_{n}-F_{2} x^{*}\right)-\left(x^{*}-y^{*}\right)\right\|^{2}\right] \\
& =\frac{1}{2}\left[\left\|\widetilde{v}_{n}-x^{*}\right\|^{2}+\left\|w_{n}-y^{*}\right\|^{2}-\left\|\left(\widetilde{v}_{n}-w_{n}\right)-\left(x^{*}-y^{*}\right)\right\|^{2}\right. \\
& \quad+2 v_{2}\left\langle\left(\widetilde{v}_{n}-w_{n}\right)-\left(x^{*}-y^{*}\right), F_{2} \widetilde{v}_{n}-F_{2} x^{*}\right\rangle \\
& \left.\quad-v_{2}^{2}\left\|F_{2} \widetilde{v}_{n}-F_{2} x^{*}\right\|^{2}\right] \\
& \leq \frac{1}{2}\left[\left\|\widetilde{v}_{n}-x^{*}\right\|^{2}+\left\|w_{n}-y^{*}\right\|^{2}-\left\|\left(\widetilde{v}_{n}-w_{n}\right)-\left(x^{*}-y^{*}\right)\right\|^{2}\right. \\
& \left.\quad+2 v_{2}\left\|\left(\widetilde{v}_{n}-w_{n}\right)-\left(x^{*}-y^{*}\right)\right\|\left\|F_{2} \widetilde{v}_{n}-F_{2} x^{*}\right\|\right],
\end{aligned}
$$


16

Journal of Function Spaces

$$
\begin{aligned}
&\left\|\widetilde{w}_{n}-x^{*}\right\|^{2} \\
&=\left\|P_{C}\left(I-v_{1} F_{1}\right) w_{n}-P_{C}\left(I-v_{1} F_{1}\right) y^{*}\right\|^{2} \\
& \leq\left\langle\left(I-v_{1} F_{1}\right) \widetilde{v}_{n}-\left(I-v_{1} F_{1}\right) \widetilde{p}, \widetilde{w}_{n}-x^{*}\right\rangle \\
&=\frac{1}{2}\left[\left\|\left(I-v_{1} F_{1}\right) w_{n}-\left(I-v_{1} F_{1}\right) y^{*}\right\|^{2}+\left\|\widetilde{w}_{n}-x^{*}\right\|^{2}\right. \\
&\left.\quad-\left\|\left(I-v_{1} F_{1}\right) w_{n}-\left(I-v_{1} F_{1}\right) y^{*}-\left(\widetilde{w}_{n}-x^{*}\right)\right\|^{2}\right] \\
& \leq \frac{1}{2}\left[\left\|w_{n}-y^{*}\right\|^{2}+\left\|\widetilde{w}_{n}-x^{*}\right\|^{2}\right. \\
& \quad-\left\|\left(w_{n}-\widetilde{w}_{n}\right)+\left(x^{*}-y^{*}\right)\right\|^{2} \\
& \quad+2 v_{1}\left\langle F_{1} w_{n}-F_{1} y^{*},\left(w_{n}-\widetilde{w}_{n}\right)+\left(x^{*}-y^{*}\right)\right\rangle \\
&\left.\quad-v_{1}^{2}\left\|F_{1} w_{n}-F_{1} y^{*}\right\|^{2}\right] \\
& \leq \frac{1}{2}\left[\left\|\widetilde{v}_{n}-x^{*}\right\|^{2}+\left\|\widetilde{w}_{n}-x^{*}\right\|^{2}\right. \\
& \quad-\left\|\left(w_{n}-\widetilde{w}_{n}\right)+\left(x^{*}-y^{*}\right)\right\|^{2} \\
&\left.+2 v_{1}\left\langle F_{1} w_{n}-F_{1} y^{*},\left(w_{n}-\widetilde{w}_{n}\right)+\left(x^{*}-y^{*}\right)\right\rangle\right] .
\end{aligned}
$$

Thus, we have

$$
\begin{aligned}
\left\|w_{n}-y^{*}\right\|^{2} \leq & \left\|\widetilde{v}_{n}-x^{*}\right\|^{2}-\left\|\left(\widetilde{v}_{n}-w_{n}\right)-\left(x^{*}-y^{*}\right)\right\|^{2} \\
& +2 v_{2}\left\|\left(\widetilde{v}_{n}-w_{n}\right)-\left(x^{*}-y^{*}\right)\right\|\left\|F_{2} \widetilde{v}_{n}-F_{2} x^{*}\right\|,
\end{aligned}
$$

$$
\begin{aligned}
\left\|\widetilde{w}_{n}-x^{*}\right\|^{2} \leq & \left\|\widetilde{v}_{n}-x^{*}\right\|^{2}-\left\|\left(w_{n}-\widetilde{w}_{n}\right)+\left(x^{*}-y^{*}\right)\right\|^{2} \\
& +2 v_{1}\left\|F_{1} w_{n}-F_{1} y^{*}\right\|\left\|\left(w_{n}-\widetilde{w}_{n}\right)+\left(x^{*}-y^{*}\right)\right\|,
\end{aligned}
$$

respectively. Consequently, from (56), (73), and (98), it follows that

$$
\begin{aligned}
\| x_{n+1}- & x^{*} \|^{2} \\
\leq & \left(1-\alpha_{n} \tau\right)\left\|G \widetilde{v}_{n}-x^{*}\right\|^{2}-2 \mu \alpha_{n}\left\langle\widetilde{A}_{2} x^{*}, x_{n+1}-x^{*}\right\rangle \\
\leq & \left\|\widetilde{w}_{n}-x^{*}\right\|^{2}+2 \mu \alpha_{n}\left\|\widetilde{A}_{2} x^{*}\right\|\left\|x_{n+1}-x^{*}\right\| \\
\leq & \left\|\widetilde{v}_{n}-x^{*}\right\|^{2}-\left\|\left(w_{n}-\widetilde{w}_{n}\right)+\left(x^{*}-y^{*}\right)\right\|^{2} \\
& +2 v_{1}\left\|F_{1} w_{n}-F_{1} y^{*}\right\|\left\|\left(w_{n}-\widetilde{w}_{n}\right)+\left(x^{*}-y^{*}\right)\right\| \\
& +2 \mu \alpha_{n}\left\|\widetilde{A}_{2} x^{*}\right\|\left\|x_{n+1}-x^{*}\right\| \\
= & \left\|v_{n}-x^{*}-\rho_{n} \widetilde{A}_{1} v_{n}\right\|^{2}-\left\|\left(w_{n}-\widetilde{w}_{n}\right)+\left(x^{*}-y^{*}\right)\right\|^{2} \\
& +2 v_{1}\left\|F_{1} w_{n}-F_{1} y^{*}\right\|\left\|\left(w_{n}-\widetilde{w}_{n}\right)+\left(x^{*}-y^{*}\right)\right\| \\
& +2 \mu \alpha_{n}\left\|\widetilde{A}_{2} x^{*}\right\|\left\|x_{n+1}-x^{*}\right\|
\end{aligned}
$$

$$
\begin{aligned}
\leq & \left\|v_{n}-x^{*}\right\|^{2}+2 \rho_{n}\left\|\widetilde{A}_{1} v_{n}\right\|\left\|\widetilde{v}_{n}-x^{*}\right\| \\
& -\left\|\left(w_{n}-\widetilde{w}_{n}\right)+\left(x^{*}-y^{*}\right)\right\|^{2} \\
& +2 v_{1}\left\|F_{1} w_{n}-F_{1} y^{*}\right\|\left\|\left(w_{n}-\widetilde{w}_{n}\right)+\left(x^{*}-y^{*}\right)\right\| \\
& +2 \mu \alpha_{n}\left\|\widetilde{A}_{2} x^{*}\right\|\left\|x_{n+1}-x^{*}\right\| \\
\leq & \left\|x_{n}-x^{*}\right\|^{2}+2 \rho_{n}\left\|\widetilde{A}_{1} v_{n}\right\|\left\|\widetilde{v}_{n}-x^{*}\right\| \\
& -\left\|\left(w_{n}-\widetilde{w}_{n}\right)+\left(x^{*}-y^{*}\right)\right\|^{2} \\
& +2 v_{1}\left\|F_{1} w_{n}-F_{1} y^{*}\right\|\left\|\left(w_{n}-\widetilde{w}_{n}\right)+\left(x^{*}-y^{*}\right)\right\| \\
& +2 \mu \alpha_{n}\left\|\widetilde{A}_{2} x^{*}\right\|\left\|x_{n+1}-x^{*}\right\|,
\end{aligned}
$$

which yields

$$
\begin{aligned}
\|\left(w_{n}-\right. & \left.\widetilde{w}_{n}\right)+\left(x^{*}-y^{*}\right) \|^{2} \\
\leq & \left\|x_{n}-x^{*}\right\|^{2}-\left\|x_{n+1}-x^{*}\right\|^{2}+2 \rho_{n}\left\|\widetilde{A}_{1} v_{n}\right\|\left\|\widetilde{v}_{n}-x^{*}\right\| \\
& +2 v_{1}\left\|F_{1} w_{n}-F_{1} y^{*}\right\|\left\|\left(w_{n}-\widetilde{w}_{n}\right)+\left(x^{*}-y^{*}\right)\right\| \\
& +2 \mu \alpha_{n}\left\|\widetilde{A}_{2} x^{*}\right\|\left\|x_{n+1}-x^{*}\right\| \\
\leq & \left\|x_{n}-x_{n+1}\right\|\left(\left\|x_{n}-x^{*}\right\|+\left\|x_{n+1}-x^{*}\right\|\right) \\
& +2 \rho_{n}\left\|\widetilde{A}_{1} v_{n}\right\|\left\|\widetilde{v}_{n}-x^{*}\right\| \\
& +2 v_{1}\left\|F_{1} w_{n}-F_{1} y^{*}\right\|\left\|\left(w_{n}-\widetilde{w}_{n}\right)+\left(x^{*}-y^{*}\right)\right\| \\
& +2 \mu \alpha_{n}\left\|\widetilde{A}_{2} x^{*}\right\|\left\|x_{n+1}-x^{*}\right\| .
\end{aligned}
$$

Since $\alpha_{n} \rightarrow 0, \rho_{n} \rightarrow 0$, and $\left\|x_{n+1}-x_{n}\right\| \rightarrow 0$ and $\left\{x_{n}\right\},\left\{v_{n}\right\}$, $\left\{\widetilde{v}_{n}\right\},\left\{w_{n}\right\}$, and $\left\{\widetilde{w}_{n}\right\}$ are bounded sequences, it follows from (95) that

$$
\lim _{n \rightarrow \infty}\left\|\left(w_{n}-\widetilde{w}_{n}\right)+\left(x^{*}-y^{*}\right)\right\|=0
$$

Also, from (56), (57), (73), and (97), it follows that

$$
\begin{aligned}
&\left\|x_{n+1}-x^{*}\right\|^{2} \\
& \leq\left(1-\alpha_{n} \tau\right)\left\|G \widetilde{v}_{n}-x^{*}\right\|^{2}-2 \mu \alpha_{n}\left\langle\widetilde{A}_{2} x^{*}, x_{n+1}-x^{*}\right\rangle \\
& \leq\left\|\widetilde{w}_{n}-x^{*}\right\|^{2}+2 \mu \alpha_{n}\left\|\widetilde{A}_{2} x^{*}\right\|\left\|x_{n+1}-x^{*}\right\| \\
& \leq\left\|w_{n}-y^{*}\right\|^{2}+2 \mu \alpha_{n}\left\|\widetilde{A}_{2} x^{*}\right\|\left\|x_{n+1}-x^{*}\right\| \\
& \leq\left\|\widetilde{v}_{n}-x^{*}\right\|^{2}-\left\|\left(\widetilde{v}_{n}-w_{n}\right)-\left(x^{*}-y^{*}\right)\right\|^{2} \\
&+2 v_{2}\left\|\left(\widetilde{v}_{n}-w_{n}\right)-\left(x^{*}-y^{*}\right)\right\|\left\|F_{2} \widetilde{v}_{n}-F_{2} x^{*}\right\|
\end{aligned}
$$




$$
\begin{aligned}
& +2 \mu \alpha_{n}\left\|\widetilde{A}_{2} x^{*}\right\|\left\|x_{n+1}-x^{*}\right\| \\
= & \left\|v_{n}-x^{*}-\rho_{n} \widetilde{A}_{1} v_{n}\right\|^{2}-\left\|\left(\widetilde{v}_{n}-w_{n}\right)-\left(x^{*}-y^{*}\right)\right\|^{2} \\
& +2 v_{2}\left\|\left(\widetilde{v}_{n}-w_{n}\right)-\left(x^{*}-y^{*}\right)\right\|\left\|F_{2} \widetilde{v}_{n}-F_{2} x^{*}\right\| \\
& +2 \mu \alpha_{n}\left\|\widetilde{A}_{2} x^{*}\right\|\left\|x_{n+1}-x^{*}\right\| \\
\leq & \left\|v_{n}-x^{*}\right\|^{2}+2 \rho_{n}\left\|\widetilde{A}_{1} v_{n}\right\|\left\|\widetilde{v}_{n}-x^{*}\right\| \\
& -\left\|\left(\widetilde{v}_{n}-w_{n}\right)-\left(x^{*}-y^{*}\right)\right\|^{2} \\
& +2 v_{2}\left\|\left(\widetilde{v}_{n}-w_{n}\right)-\left(x^{*}-y^{*}\right)\right\|\left\|F_{2} \widetilde{v}_{n}-F_{2} x^{*}\right\| \\
& +2 \mu \alpha_{n}\left\|\widetilde{A}_{2} x^{*}\right\|\left\|x_{n+1}-x^{*}\right\| \\
\leq & \left\|x_{n}-x^{*}\right\|^{2}+2 \rho_{n}\left\|\widetilde{A}_{1} v_{n}\right\|\left\|\widetilde{v}_{n}-x^{*}\right\| \\
& -\left\|\left(\widetilde{v}_{n}-w_{n}\right)-\left(x^{*}-y^{*}\right)\right\|^{2} \\
& +2 v_{2}\left\|\left(\widetilde{v}_{n}-w_{n}\right)-\left(x^{*}-y^{*}\right)\right\|\left\|F_{2} \widetilde{v}_{n}-F_{2} x^{*}\right\| \\
& +2 \mu \alpha_{n}\left\|\widetilde{A}_{2} x^{*}\right\|\left\|x_{n+1}-x^{*}\right\|,
\end{aligned}
$$

which leads to

$$
\begin{aligned}
&\left\|\left(\widetilde{v}_{n}-w_{n}\right)-\left(x^{*}-y^{*}\right)\right\|^{2} \\
& \leq\left\|x_{n}-x^{*}\right\|^{2}-\left\|x_{n+1}-x^{*}\right\|^{2}+2 \rho_{n}\left\|\widetilde{A}_{1} v_{n}\right\|\left\|\widetilde{v}_{n}-x^{*}\right\| \\
&+2 v_{2}\left\|\left(\widetilde{v}_{n}-w_{n}\right)-\left(x^{*}-y^{*}\right)\right\|\left\|F_{2} \widetilde{v}_{n}-F_{2} x^{*}\right\| \\
&+2 \mu \alpha_{n}\left\|\widetilde{A}_{2} x^{*}\right\|\left\|x_{n+1}-x^{*}\right\| \\
& \leq\left\|x_{n}-x_{n+1}\right\|\left(\left\|x_{n}-x^{*}\right\|+\left\|x_{n+1}-x^{*}\right\|\right) \\
&+2 \rho_{n}\left\|\widetilde{A}_{1} v_{n}\right\|\left\|\widetilde{v}_{n}-x^{*}\right\| \\
&+2 v_{2}\left\|\left(\widetilde{v}_{n}-w_{n}\right)-\left(x^{*}-y^{*}\right)\right\|\left\|F_{2} \widetilde{v}_{n}-F_{2} x^{*}\right\| \\
&+2 \mu \alpha_{n}\left\|\widetilde{A}_{2} x^{*}\right\|\left\|x_{n+1}-x^{*}\right\| .
\end{aligned}
$$

Since $\alpha_{n} \rightarrow 0, \rho_{n} \rightarrow 0$, and $\left\|x_{n+1}-x_{n}\right\| \rightarrow 0$ and $\left\{x_{n}\right\}$, $\left\{v_{n}\right\},\left\{\widetilde{v}_{n}\right\}$, and $\left\{w_{n}\right\}$ are bounded sequences, it follows from (95) that

$$
\lim _{n \rightarrow \infty}\left\|\left(\widetilde{v}_{n}-w_{n}\right)-\left(x^{*}-y^{*}\right)\right\|=0 .
$$

Note that

$$
\begin{aligned}
\left\|\widetilde{v}_{n}-\widetilde{w}_{n}\right\| \leq & \left\|\left(\widetilde{v}_{n}-w_{n}\right)-\left(x^{*}-y^{*}\right)\right\| \\
& +\left\|\left(w_{n}-\widetilde{w}_{n}\right)+\left(x^{*}-y^{*}\right)\right\| .
\end{aligned}
$$

Hence, from (101) and (104), we get

$$
\lim _{n \rightarrow \infty}\left\|\widetilde{v}_{n}-G \widetilde{v}_{n}\right\|=\lim _{n \rightarrow \infty}\left\|\widetilde{v}_{n}-\widetilde{w}_{n}\right\|=0 .
$$

Also, observe that $\left\|\widetilde{v}_{n}-x_{n}\right\| \leq\left\|v_{n}-x_{n}\right\|+\rho_{n}\left\|\widetilde{A}_{1} v_{n}\right\|$ and

$$
\left\|y_{n}-x_{n}\right\| \leq\left\|x_{n+1}-x_{n}\right\|+\mu \alpha_{n}\left\|\widetilde{A}_{2} y_{n}\right\| \text {. }
$$

Hence, from (91), $\alpha_{n} \rightarrow 0, \rho_{n} \rightarrow 0$, and $\left\|x_{n+1}-x_{n}\right\| \rightarrow 0$, we obtain that

$$
\lim _{n \rightarrow \infty}\left\|\widetilde{v}_{n}-x_{n}\right\|=0, \quad \lim _{n \rightarrow \infty}\left\|y_{n}-x_{n}\right\|=0 .
$$

So, from (106) and (108), we deduce that

$$
\begin{aligned}
& \left\|W_{n} \widetilde{v}_{n}-\widetilde{v}_{n}\right\| \\
& \leq\left\|W_{n} \widetilde{v}_{n}-y_{n}\right\|+\left\|y_{n}-x_{n}\right\|+\left\|x_{n}-\widetilde{v}_{n}\right\| \\
& =\left\|W_{n} \widetilde{v}_{n}-W_{n} G \widetilde{v}_{n}\right\|+\left\|y_{n}-x_{n}\right\|+\left\|x_{n}-\widetilde{v}_{n}\right\| \\
& \leq\left\|\widetilde{v}_{n}-G \widetilde{v}_{n}\right\|+\left\|y_{n}-x_{n}\right\|+\left\|x_{n}-\widetilde{v}_{n}\right\| \longrightarrow 0 \\
& \quad \text { as } n \longrightarrow \infty .
\end{aligned}
$$

In addition, it is clear that

$$
\left\|\widetilde{v}_{n}-W \widetilde{v}_{n}\right\| \leq\left\|\widetilde{v}_{n}-W_{n} \widetilde{v}_{n}\right\|+\left\|W_{n} \widetilde{v}_{n}-W \widetilde{v}_{n}\right\| .
$$

Thus, we conclude from [24, Remark 3.2], (3.28,) and the boundedness of $\left\{\widetilde{v}_{n}\right\}$ that

$$
\lim _{n \rightarrow \infty}\left\|\widetilde{v}_{n}-W \widetilde{v}_{n}\right\|=0
$$

Step 4. We prove that $\omega_{w}\left(x_{n}\right) \subset \Omega$.

Indeed, since $H$ is reflexive and $\left\{x_{n}\right\}$ is bounded, there exists at least a weak convergence subsequence of $\left\{x_{n}\right\}$. Hence, it is known that $\omega_{w}\left(x_{n}\right) \neq \emptyset$. Now, take an arbitrary $w \in$ $\omega_{w}\left(x_{n}\right)$. Then, there exists a subsequence $\left\{x_{n}\right\}$ of $\left\{x_{n}\right\}$ such that $x_{n_{i}} \rightarrow w$. From (85)-(89), (91), and (108), we have that $u_{n_{i}} \rightarrow w, v_{n_{i}} \rightarrow w, \widetilde{v}_{n_{i}} \rightarrow w, \Lambda_{n_{i}}^{m} u_{n_{i}} \rightarrow w$, and $\Delta_{n_{i}}^{k} x_{n_{i}} \rightarrow w$, where $m \in\{1,2, \ldots, N\}$ and $k \in\{1,2, \ldots, M\}$. Utilizing Lemma 6, we deduce from $\widetilde{v}_{n_{i}} \rightarrow w$, (106), and (111) that $w \in \operatorname{GSVI}(G)$ and $w \in \operatorname{Fix}(W)=\bigcap_{n=1}^{\infty} \operatorname{Fix}\left(T_{n}\right)$ (due to Lemma 8). Next, we prove that $w \in \bigcap_{m=1}^{N} I\left(B_{m}, R_{m}\right)$. As a matter of fact, since $B_{m}$ is $\eta_{m}$-inverse strongly monotone, $B_{m}$ is a monotone and Lipschitz continuous mapping. It follows from Lemma 17 that $R_{m}+B_{m}$ is maximal monotone. Let $(v, g) \in G\left(R_{m}+B_{m}\right)$; that is, $g-B_{m} v \in R_{m} v$. Again, since $\Lambda_{n}^{m} u_{n}=J_{R_{m}, \lambda_{m, n}}\left(I-\lambda_{m, n} B_{m}\right) \Lambda_{n}^{m-1} u_{n}, n \geq 1, m \in\{1,2, \ldots, N\}$, we have

$$
\Lambda_{n}^{m-1} u_{n}-\lambda_{m, n} B_{m} \Lambda_{n}^{m-1} u_{n} \in\left(I+\lambda_{m, n} R_{m}\right) \Lambda_{n}^{m} u_{n} .
$$

That is,

$$
\frac{1}{\lambda_{m, n}}\left(\Lambda_{n}^{m-1} u_{n}-\Lambda_{n}^{m} u_{n}-\lambda_{m, n} B_{m} \Lambda_{n}^{m-1} u_{n}\right) \in R_{m} \Lambda_{n}^{m} u_{n} .
$$

In terms of the monotonicity of $R_{m}$, we get

$$
\begin{aligned}
& \left\langle v-\Lambda_{n}^{m} u_{n}, g-B_{m} v\right. \\
& \left.\quad-\frac{1}{\lambda_{m, n}}\left(\Lambda_{n}^{m-1} u_{n}-\Lambda_{n}^{m} u_{n}-\lambda_{m, n} B_{m} \Lambda_{n}^{m-1} u_{n}\right)\right\rangle \geq 0
\end{aligned}
$$


and hence

$$
\begin{aligned}
\langle v- & \left.\Lambda_{n}^{m} u_{n}, g\right\rangle \\
\geq & \left\langle v-\Lambda_{n}^{m} u_{n}, B_{m} v\right. \\
& \left.+\frac{1}{\lambda_{m, n}}\left(\Lambda_{n}^{m-1} u_{n}-\Lambda_{n}^{m} u_{n}-\lambda_{m, n} B_{m} \Lambda_{n}^{m-1} u_{n}\right)\right\rangle \\
= & \left\langle v-\Lambda_{n}^{m} u_{n}, B_{m} v-B_{m} \Lambda_{n}^{m} u_{n}+B_{m} \Lambda_{n}^{m} u_{n}\right. \\
& \left.-B_{m} \Lambda_{n}^{m-1} u_{n}+\frac{1}{\lambda_{m, n}}\left(\Lambda_{n}^{m-1} u_{n}-\Lambda_{n}^{m} u_{n}\right)\right\rangle \\
\geq & \left\langle v-\Lambda_{n}^{m} u_{n}, B_{m} \Lambda_{n}^{m} u_{n}-B_{m} \Lambda_{n}^{m-1} u_{n}\right\rangle \\
& +\left\langle v-\Lambda_{n}^{m} u_{n}, \frac{1}{\lambda_{m, n}}\left(\Lambda_{n}^{m-1} u_{n}-\Lambda_{n}^{m} u_{n}\right)\right\rangle
\end{aligned}
$$

In particular,

$$
\begin{aligned}
& \left\langle v-\Lambda_{n_{i}}^{m} u_{n_{i}}, g\right\rangle \\
& \geq\left\langle v-\Lambda_{n_{i}}^{m} u_{n_{i}}, B_{m} \Lambda_{n_{i}}^{m} u_{n_{i}}-B_{m} \Lambda_{n_{i}}^{m-1} u_{n_{i}}\right\rangle \\
& \quad+\left\langle v-\Lambda_{n_{i}}^{m} u_{n_{i}}, \frac{1}{\lambda_{m, n_{i}}}\left(\Lambda_{n_{i}}^{m-1} u_{n_{i}}-\Lambda_{n_{i}}^{m} u_{n_{i}}\right)\right\rangle .
\end{aligned}
$$

Since $\left\|\Lambda_{n}^{m} u_{n}-\Lambda_{n}^{m-1} u_{n}\right\| \rightarrow 0$ (due to (85)) and $\| B_{m} \Lambda_{n}^{m} u_{n}-$ $B_{m} \Lambda_{n}^{m-1} u_{n} \| \rightarrow 0$ (due to the Lipschitz continuity of $B_{m}$ ), we conclude from $\Lambda_{n_{i}}^{m} u_{n_{i}} \rightarrow w$ and $\left\{\lambda_{i, n}\right\} \subset\left[a_{i}, b_{i}\right] \subset\left(0,2 \eta_{i}\right)$ that

$$
\lim _{i \rightarrow \infty}\left\langle v-\Lambda_{n_{i}}^{m} u_{n_{i}}, g\right\rangle=\langle v-w, g\rangle \geq 0
$$

It follows from the maximal monotonicity of $B_{m}+R_{m}$ that $0 \in\left(R_{m}+B_{m}\right) w$; that is, $w \in I\left(B_{m}, R_{m}\right)$. Therefore, $w \in \bigcap_{m=1}^{N} I\left(B_{m}, R_{m}\right)$. Next, we prove that $w \in \bigcap_{k=1}^{M} \operatorname{GMEP}\left(\Theta_{k}, \varphi_{k}, A_{k}\right)$. Since $\Delta_{n}^{k} x_{n}=T_{r_{k, n}}^{\left(\Theta_{k}, \varphi_{k}\right)}(I-$ $\left.r_{k, n} A_{k}\right) \Delta_{n}^{k-1} x_{n}, n \geq 1, k \in\{1,2, \ldots, M\}$, we have

$$
\begin{aligned}
& \Theta_{k}\left(\Delta_{n}^{k} x_{n}, y\right)+\varphi_{k}(y)-\varphi_{k}\left(\Delta_{n}^{k} x_{n}\right) \\
& +\left\langle A_{k} \Delta_{n}^{k-1} x_{n}, y-\Delta_{n}^{k} x_{n}\right\rangle \\
& \quad+\frac{1}{r_{k, n}}\left\langle y-\Delta_{n}^{k} x_{n}, \Delta_{n}^{k} x_{n}-\Delta_{n}^{k-1} x_{n}\right\rangle \geq 0 .
\end{aligned}
$$

By (A2), we have

$$
\begin{aligned}
& \varphi_{k}(y)-\varphi_{k}\left(\Delta_{n}^{k} x_{n}\right)+\left\langle A_{k} \Delta_{n}^{k-1} x_{n}, y-\Delta_{n}^{k} x_{n}\right\rangle \\
& \quad+\frac{1}{r_{k, n}}\left\langle y-\Delta_{n}^{k} x_{n}, \Delta_{n}^{k} x_{n}-\Delta_{n}^{k-1} x_{n}\right\rangle \geq \Theta_{k}\left(y, \Delta_{n}^{k} x_{n}\right) .
\end{aligned}
$$

Let $z_{t}=t y+(1-t) w$ for all $t \in(0,1]$ and $y \in C$. This implies that $z_{t} \in C$. Then, we have

$$
\begin{aligned}
\left\langle z_{t}-\right. & \left.\Delta_{n}^{k} x_{n}, A_{k} z_{t}\right\rangle \\
\geq & \varphi_{k}\left(\Delta_{n}^{k} x_{n}\right)-\varphi_{k}\left(z_{t}\right)+\left\langle z_{t}-\Delta_{n}^{k} x_{n}, A_{k} z_{t}\right\rangle \\
& -\left\langle z_{t}-\Delta_{n}^{k} x_{n}, A_{k} \Delta_{n}^{k-1} x_{n}\right\rangle \\
& -\left\langle z_{t}-\Delta_{n}^{k} x_{n}, \frac{\Delta_{n}^{k} x_{n}-\Delta_{n}^{k-1} x_{n}}{r_{k, n}}\right\rangle+\Theta_{k}\left(z_{t}, \Delta_{n}^{k} x_{n}\right) \\
= & \varphi_{k}\left(\Delta_{n}^{k} x_{n}\right)-\varphi_{k}\left(z_{t}\right)+\left\langle z_{t}-\Delta_{n}^{k} x_{n}, A_{k} z_{t}-A_{k} \Delta_{n}^{k} x_{n}\right\rangle \\
& +\left\langle z_{t}-\Delta_{n}^{k} x_{n}, A_{k} \Delta_{n}^{k} x_{n}-A_{k} \Delta_{n}^{k-1} x_{n}\right\rangle \\
& -\left\langle z_{t}-\Delta_{n}^{k} x_{n}, \frac{\Delta_{n}^{k} x_{n}-\Delta_{n}^{k-1} x_{n}}{r_{k, n}}\right\rangle+\Theta_{k}\left(z_{t}, \Delta_{n}^{k} x_{n}\right)
\end{aligned}
$$

By (88), we have $\left\|A_{k} \Delta_{n}^{k} x_{n}-A_{k} \Delta_{n}^{k-1} x_{n}\right\| \rightarrow 0$ as $n \rightarrow \infty$. Furthermore, by the monotonicity of $A_{k}$, we obtain $\left\langle z_{t}-\right.$ $\left.\Delta_{n}^{k} x_{n}, A_{k} z_{t}-A_{k} \Delta_{n}^{k} x_{n}\right\rangle \geq 0$. Then, by (A4), we obtain

$$
\left\langle z_{t}-w, A_{k} z_{t}\right\rangle \geq \varphi_{k}(w)-\varphi_{k}\left(z_{t}\right)+\Theta_{k}\left(z_{t}, w\right) .
$$

Utilizing (A1), (A4), and (121), we obtain

$$
\begin{aligned}
0= & \Theta_{k}\left(z_{t}, z_{t}\right)+\varphi_{k}\left(z_{t}\right)-\varphi_{k}\left(z_{t}\right) \\
\leq & t \Theta_{k}\left(z_{t}, y\right)+(1-t) \Theta_{k}\left(z_{t}, w\right) \\
& +t \varphi_{k}(y)+(1-t) \varphi_{k}(w)-\varphi_{k}\left(z_{t}\right) \\
\leq & t\left[\Theta_{k}\left(z_{t}, y\right)+\varphi_{k}(y)-\varphi_{k}\left(z_{t}\right)\right]+(1-t)\left\langle z_{t}-w, A_{k} z_{t}\right\rangle \\
= & t\left[\Theta_{k}\left(z_{t}, y\right)+\varphi_{k}(y)-\varphi_{k}\left(z_{t}\right)\right] \\
& +(1-t) t\left\langle y-w, A_{k} z_{t}\right\rangle,
\end{aligned}
$$

and hence

$$
0 \leq \Theta_{k}\left(z_{t}, y\right)+\varphi_{k}(y)-\varphi_{k}\left(z_{t}\right)+(1-t)\left\langle y-w, A_{k} z_{t}\right\rangle .
$$

Letting $t \rightarrow 0$, we have, for each $y \in C$,

$$
0 \leq \Theta_{k}(w, y)+\varphi_{k}(y)-\varphi_{k}(w)+\left\langle y-w, A_{k} w\right\rangle .
$$

This implies that $w \in \operatorname{GMEP}\left(\Theta_{k}, \varphi_{k}, A_{k}\right)$ and hence $w \in \bigcap_{k=1}^{M} \operatorname{GMEP}\left(\Theta_{k}, \varphi_{k}, A_{k}\right)$. Thus, $w \in \Omega=$ $\bigcap_{n=1}^{\infty} \operatorname{Fix}\left(T_{n}\right) \cap \bigcap_{k=1}^{M} \operatorname{GMEP}\left(\Theta_{k}, \varphi_{k}, A_{k}\right) \cap \bigcap_{m=1}^{N} I\left(B_{m}, R_{m}\right)$. Consequently, $w \in \bigcap_{n=1}^{\infty} \operatorname{Fix}\left(T_{n}\right) \cap \bigcap_{k=1}^{M} \operatorname{GMEP}\left(\Theta_{k}, \varphi_{k}, A_{k}\right) \cap$ $\bigcap_{m=1}^{N} I\left(B_{m}, R_{m}\right) \cap \operatorname{GSVI}(G)=: \Omega$. This shows that $\omega_{w}\left(x_{n}\right) \subset$ $\Omega$.

Step 5. We prove that $\omega_{w}\left(x_{n}\right) \subset \operatorname{VI}\left(\Omega, \widetilde{A}_{1}\right)$ provided $\| x_{n}-$ $y_{n} \|=o\left(\rho_{n}\right)$ additionally. 
Indeed, take an arbitrary $w \in \omega_{w}\left(x_{n}\right)$. Then, there exists a subsequence $\left\{x_{n_{i}}\right\}$ of $\left\{x_{n}\right\}$ such that $x_{n_{i}} \rightarrow w$. Utilizing the arguments similar to those of (56) and (57), we can readily obtain that for all $p \in \Omega$

$$
\left\|v_{n}-p\right\| \leq\left\|x_{n}-p\right\|, \quad\left\|G \widetilde{v}_{n}-p\right\| \leq\left\|\widetilde{v}_{n}-p\right\| .
$$

Since $A_{1}$ is $\alpha$-inverse strongly monotone, from (48) and (125) we conclude that for all $p \in \Omega$

$$
\begin{aligned}
\left\|y_{n}-p\right\|^{2} & \\
= & \left\|W_{n} G \widetilde{v}_{n}-p\right\|^{2} \\
\leq & \left\|\widetilde{v}_{n}-p\right\|^{2} \\
= & \left\|v_{n}-p-\rho_{n} \widetilde{A}_{1} v_{n}\right\|^{2} \\
\leq & \left\|v_{n}-p\right\|^{2}-2 \rho_{n}\left\langle\widetilde{A}_{1} v_{n}, \widetilde{v}_{n}-p\right\rangle \\
= & \left\|v_{n}-p\right\|^{2}-2 \rho_{n}\left(\left\langle\widetilde{A}_{1} v_{n}, v_{n}-p\right\rangle+\left\langle\widetilde{A}_{1} v_{n}, \widetilde{v}_{n}-v_{n}\right\rangle\right) \\
= & \left\|v_{n}-p\right\|^{2}-2 \rho_{n}\left(\left\langle\widetilde{A}_{1} v_{n}-\widetilde{A}_{1} p, v_{n}-p\right\rangle\right. \\
\quad & \left.\quad+\left\langle\widetilde{A}_{1} p, v_{n}-p\right\rangle+\left\langle\widetilde{A}_{1} v_{n}, \widetilde{v}_{n}-v_{n}\right\rangle\right) \\
\leq & \left\|x_{n}-p\right\|^{2}-2 \rho_{n}\left\langle\widetilde{A}_{1} p, v_{n}-p\right\rangle \\
& +2 \rho_{n}\left\|\widetilde{A}_{1} v_{n}\right\|\left\|\widetilde{v}_{n}-v_{n}\right\|,
\end{aligned}
$$

which implies that

$$
\begin{aligned}
& \left\langle\widetilde{A}_{1} p, v_{n}-p\right\rangle \\
& \quad \leq \frac{1}{2 \rho_{n}}\left(\left\|x_{n}-p\right\|^{2}-\left\|y_{n}-p\right\|^{2}\right)+\left\|\widetilde{A}_{1} v_{n}\right\|\left\|\widetilde{v}_{n}-v_{n}\right\| \\
& \quad \leq \frac{\left\|x_{n}-y_{n}\right\|}{2 \rho_{n}}\left(\left\|x_{n}-p\right\|+\left\|y_{n}-p\right\|\right)+\rho_{n}\left\|\widetilde{A}_{1} v_{n}\right\|^{2} .
\end{aligned}
$$

So, from $\rho_{n} \rightarrow 0$ and the assumption $\left\|x_{n}-y_{n}\right\|=o\left(\rho_{n}\right)$, we get

$$
\limsup _{n \rightarrow \infty}\left\langle\widetilde{A}_{1} p, v_{n}-p\right\rangle \leq 0 .
$$

Thus, it follows from (91) that for all $p \in \Omega$

$$
\begin{aligned}
\left\langle\widetilde{A}_{1} p, w-p\right\rangle & =\lim _{i \rightarrow \infty}\left\langle\widetilde{A}_{1} p, x_{n_{i}}-p\right\rangle \\
& \leq \limsup _{n \rightarrow \infty}\left\langle\widetilde{A}_{1} p, x_{n}-p\right\rangle \\
& =\limsup _{n \rightarrow \infty}\left(\left\langle\widetilde{A}_{1} p, v_{n}-p\right\rangle+\left\langle\widetilde{A}_{1} p, x_{n}-v_{n}\right\rangle\right) \\
& =\limsup _{n \rightarrow \infty}\left\langle\widetilde{A}_{1} p, v_{n}-p\right\rangle \\
& \leq 0 .
\end{aligned}
$$

That is,

$$
\left\langle\widetilde{A}_{1} p, p-w\right\rangle \geq 0, \quad \forall p \in \Omega \text {. }
$$

Since $\widetilde{A}_{1}$ is $\alpha$-inverse strongly monotone, by Minty's Lemma [16], we know that (130) is equivalent to the VIP

$$
\left\langle\widetilde{A}_{1} w, p-w\right\rangle \geq 0, \quad \forall p \in \Omega .
$$

This shows that $w \in \operatorname{VI}\left(\Omega, \widetilde{A}_{1}\right)$. Therefore, $\omega_{w}\left(x_{n}\right) \quad c$ $\operatorname{VI}\left(\Omega, \widetilde{A}_{1}\right)$.

Theorem 19. Assume that there hold all the conditions in Theorem 18. Then, we have

(i) $\left\{x_{n}\right\}$ converges strongly to a point $u^{*} \in \Omega$, which is a unique solution of the VIP

$$
\left\langle\widetilde{A}_{2} u^{*}, p-u^{*}\right\rangle \geq 0, \quad \forall p \in \Omega ;
$$

(ii) $\left\{x_{n}\right\}$ converges strongly to a unique solution of THVI (19) provided $\left\|x_{n}-y_{n}\right\|=o\left(\theta_{n}\right)$ additionally.

Proof. Since $\widetilde{A}_{2}$ is $\beta$-strongly monotone and L-Lipschitz continuous, there exists a unique solution $u^{*} \in \Omega$ of the VIP

$$
\left\langle\widetilde{A_{2}} u^{*}, p-u^{*}\right\rangle \geq 0, \quad \forall p \in \Omega .
$$

Now, let us show that

$$
\limsup _{n \rightarrow \infty}\left\langle\widetilde{A}_{2} u^{*}, u^{*}-x_{n}\right\rangle \leq 0 .
$$

Since $\left\{x_{n}\right\}$ is bounded, we may assume, without loss of generality, that there exists a subsequence $\left\{x_{n_{i}}\right\}$ of $\left\{x_{n}\right\}$ such that $x_{n_{i}} \rightarrow w$ and

$$
\begin{aligned}
\limsup _{n \rightarrow \infty} & \left\langle\widetilde{A}_{2} u^{*}, u^{*}-x_{n}\right\rangle \\
& =\lim _{i \rightarrow \infty}\left\langle\widetilde{A}_{2} u^{*}, u^{*}-x_{n_{i}}\right\rangle=\left\langle\widetilde{A}_{2} u^{*}, u^{*}-w\right\rangle .
\end{aligned}
$$

In terms of Theorem 18 (ii), we know that $w \in \omega_{w}\left(x_{n}\right) \subset \Omega$. So, from (133), it follows that

$$
\limsup _{n \rightarrow \infty}\left\langle\widetilde{A}_{2} u^{*}, u^{*}-x_{n}\right\rangle=\left\langle\widetilde{A}_{2} u^{*}, u^{*}-w\right\rangle \leq 0 .
$$

Next, let us show that $\lim _{n \rightarrow \infty}\left\|x_{n}-u^{*}\right\|=0$. In fact, utilizing Lemma 4, from (48) and (125) with $p=u^{*}$, we get

$$
\begin{aligned}
\| x_{n+1}- & u^{*} \|^{2} \\
= & \left\|\left(I-\mu \alpha_{n} \widetilde{A}_{2}\right) W_{n} G \widetilde{v}_{n}-u^{*}\right\|^{2} \\
= & \|\left(I-\mu \alpha_{n} \widetilde{A}_{2}\right) W_{n} G \widetilde{v}_{n}-\left(I-\mu \alpha_{n} \widetilde{A}_{2}\right) W_{n} G u^{*} \\
& +\left(I-\mu \alpha_{n} \widetilde{A}_{2}\right) W_{n} G u^{*}-W_{n} G u^{*} \|^{2}
\end{aligned}
$$




$$
\begin{aligned}
\leq & \left\|\left(I-\mu \alpha_{n} \widetilde{A}_{2}\right) W_{n} G \widetilde{v}_{n}-\left(I-\mu \alpha_{n} \widetilde{A}_{2}\right) W_{n} G u^{*}\right\|^{2} \\
& -2 \mu \alpha_{n}\left\langle\widetilde{A}_{2} u^{*}, x_{n+1}-u^{*}\right\rangle \\
\leq & \left(1-\alpha_{n} \tau\right)\left\|G \widetilde{v}_{n}-u^{*}\right\|^{2}-2 \mu \alpha_{n}\left\langle\widetilde{A}_{2} u^{*}, x_{n+1}-u^{*}\right\rangle \\
\leq & \left(1-\alpha_{n} \tau\right)\left\|\widetilde{v}_{n}-u^{*}\right\|^{2}-2 \mu \alpha_{n}\left\langle\widetilde{A}_{2} u^{*}, x_{n+1}-u^{*}\right\rangle \\
= & \left(1-\alpha_{n} \tau\right)\left\|v_{n}-u^{*}-\rho_{n} \widetilde{A}_{1} v_{n}\right\|^{2} \\
& -2 \mu \alpha_{n}\left\langle\widetilde{A}_{2} u^{*}, x_{n+1}-u^{*}\right\rangle \\
\leq & \left(1-\alpha_{n} \tau\right)\left(\left\|v_{n}-u^{*}\right\|^{2}-2 \rho_{n}\left\langle\widetilde{A}_{1} v_{n}, \widetilde{v}_{n}-u^{*}\right\rangle\right) \\
& -2 \mu \alpha_{n}\left\langle\widetilde{A}_{2} u^{*}, x_{n+1}-u^{*}\right\rangle \\
\leq & \left(1-\alpha_{n} \tau\right)\left\|x_{n}-u^{*}\right\|^{2}+2 \rho_{n}\left\|\widetilde{A}_{1} v_{n}\right\|\left\|\widetilde{v}_{n}-u^{*}\right\| \\
& +2 \mu \alpha_{n}\left\langle\widetilde{A}_{2} u^{*}, u^{*}-x_{n+1}\right\rangle \\
& +\alpha_{n} \tau \cdot \frac{2}{\tau}\left\{\frac{\rho_{n}}{\alpha_{n}}\left\|\widetilde{A}_{1} v_{n}\right\|\left\|\widetilde{v}_{n}-u^{*}\right\|\right. \\
= & \left(1-\alpha_{n} \tau\right)\left\|x_{n}-u^{*}\right\|^{2} \\
& \left.\left.+\widetilde{A}_{2} u^{*}, u^{*}-x_{n+1}\right\rangle\right\}, \\
& \\
& \\
& \\
&
\end{aligned}
$$

where $\tau=1-\sqrt{1-\mu\left(2 \beta-\mu L^{2}\right)}$. Since $\sum_{n=1}^{\infty} \alpha_{n}=\infty$, $\lim _{n \rightarrow \infty}\left(\rho_{n} / \alpha_{n}\right)=0$, and $\lim \sup _{n \rightarrow \infty}\left\langle\widetilde{A}_{2} u^{*}, u^{*}-x_{n+1}\right\rangle \leq 0$ (due to (136)), we deduce that $\sum_{n=1}^{\infty} \alpha_{n} \tau=\infty$ and

$$
\begin{aligned}
& \limsup _{n \rightarrow \infty} \frac{2}{\tau}\left\{\frac{\rho_{n}}{\alpha_{n}}\left\|\widetilde{A}_{1} v_{n}\right\|\left\|\widetilde{v}_{n}-u^{*}\right\|+\mu\left\langle\widetilde{A}_{2} u^{*}, u^{*}-x_{n+1}\right\rangle\right\} \\
& \leq 0
\end{aligned}
$$

Therefore, applying Lemma 10 to (137), we infer that $\lim _{n \rightarrow \infty}\left\|x_{n}-u^{*}\right\|=0$.

Finally, we prove that $\lim _{n \rightarrow \infty}\left\|x_{n}-x^{*}\right\|=0$ provided $\left\|x_{n}-y_{n}\right\|=o\left(\alpha_{n}\right)$ additionally, where $\left\{x^{*}\right\}=$ $\operatorname{VI}\left(\mathrm{VI}\left(\Omega, \widetilde{A}_{1}\right), \widetilde{A}_{2}\right)$.

Indeed, first of all, let us show that $\omega_{w}\left(x_{n}\right)=\left\{x^{*}\right\}$. As a matter of fact, take an arbitrary $w \in \omega_{w}\left(x_{n}\right)$. Then, there exists a subsequence $\left\{x_{n_{j}}\right\}$ of $\left\{x_{n}\right\}$ such that $x_{n_{j}} \rightarrow w$. Moreover, by Theorem 18 (iii), we know that $w \in \omega_{w}\left(x_{n}\right) \subset \mathrm{VI}\left(\Omega, \widetilde{A_{1}}\right)$. Utilizing the arguments similar to those of (56) and (57), we can readily obtain that for all $p \in \operatorname{VI}\left(\Omega, \widetilde{A_{1}}\right)$

$$
\left\|v_{n}-p\right\| \leq\left\|x_{n}-p\right\|, \quad\left\|G \widetilde{v}_{n}-p\right\| \leq\left\|\widetilde{v}_{n}-p\right\|
$$

Utilizing Lemma 4, from (48) and (139), we deduce that for all $p \in \operatorname{VI}\left(\Omega, \widetilde{A_{1}}\right)$

$$
\begin{aligned}
& \left\|x_{n+1}-p\right\|^{2} \\
& =\left\|\left(I-\mu \alpha_{n} \widetilde{A}_{2}\right) W_{n} G \widetilde{v}_{n}-p\right\|^{2} \\
& =\|\left(I-\mu \alpha_{n} \widetilde{A}_{2}\right) W_{n} G \widetilde{v}_{n}-\left(I-\mu \alpha_{n} \widetilde{A}_{2}\right) W_{n} G p \\
& +\left(I-\mu \alpha_{n} \widetilde{A}_{2}\right) W_{n} G p-W_{n} G p \|^{2} \\
& \leq\left\|\left(I-\mu \alpha_{n} \widetilde{A}_{2}\right) W_{n} G \widetilde{v}_{n}-\left(I-\mu \alpha_{n} \widetilde{A}_{2}\right) W_{n} G p\right\|^{2} \\
& -2 \mu \alpha_{n}\left\langle\widetilde{A}_{2} p, x_{n+1}-p\right\rangle \\
& \leq\left(1-\alpha_{n} \tau\right)\left\|G \widetilde{v}_{n}-p\right\|^{2}-2 \mu \alpha_{n}\left\langle\widetilde{A}_{2} p, x_{n+1}-p\right\rangle \\
& \leq\left(1-\alpha_{n} \tau\right)\left\|\widetilde{v}_{n}-p\right\|^{2}-2 \mu \alpha_{n}\left\langle\widetilde{A}_{2} p, x_{n+1}-p\right\rangle \\
& =\left(1-\alpha_{n} \tau\right)\left\|v_{n}-p-\rho_{n} \widetilde{A}_{1} v_{n}\right\|^{2}-2 \mu \alpha_{n}\left\langle\widetilde{A}_{2} p, x_{n+1}-p\right\rangle \\
& \leq\left(1-\alpha_{n} \tau\right)\left(\left\|v_{n}-p\right\|^{2}-2 \rho_{n}\left\langle\widetilde{A}_{1} v_{n}, \widetilde{v}_{n}-p\right\rangle\right) \\
& -2 \mu \alpha_{n}\left\langle\widetilde{A}_{2} p, x_{n+1}-p\right\rangle \\
& \leq\left\|v_{n}-p\right\|^{2}-2\left(1-\alpha_{n} \tau\right) \rho_{n}\left\langle\widetilde{A}_{1} v_{n}, \widetilde{v}_{n}-p\right\rangle \\
& -2 \mu \alpha_{n}\left\langle\widetilde{A}_{2} p, x_{n+1}-p\right\rangle \\
& \leq\left\|x_{n}-p\right\|^{2}-2\left(1-\alpha_{n} \tau\right) \rho_{n}\left\langle\widetilde{A}_{1} v_{n}, \widetilde{v}_{n}-p\right\rangle \\
& -2 \mu \alpha_{n}\left\langle\widetilde{A}_{2} p, x_{n+1}-p\right\rangle,
\end{aligned}
$$

where $\tau=1-\sqrt{1-\mu\left(2 \beta-\mu L^{2}\right)}$. So, it follows that

$$
\begin{aligned}
\left\langle\widetilde{A}_{2} p, x_{n+1}-p\right\rangle & \\
\leq & \frac{1}{2 \mu \alpha_{n}}\left(\left\|x_{n}-p\right\|^{2}-\left\|x_{n+1}-p\right\|^{2}\right) \\
& -\frac{\left(1-\alpha_{n} \tau\right) \rho_{n}}{\mu \alpha_{n}}\left\langle\widetilde{A}_{1} v_{n}, \widetilde{v}_{n}-p\right\rangle \\
\leq & \frac{\left\|x_{n}-x_{n+1}\right\|}{2 \mu \alpha_{n}}\left(\left\|x_{n}-p\right\|+\left\|x_{n+1}-p\right\|\right) \\
& +\frac{\left(1-\alpha_{n} \tau\right) \rho_{n}}{\mu \alpha_{n}}\left\|\widetilde{A}_{1} v_{n}\right\|\left\|\widetilde{v}_{n}-p\right\| .
\end{aligned}
$$

Since $\alpha_{n} \rightarrow 0, \rho_{n}=o\left(\alpha_{n}\right)$, and $\left\|x_{n+1}-x_{n}\right\|=o\left(\rho_{n}\right)$, we find that

$$
\lim _{n \rightarrow \infty} \frac{\left\|x_{n+1}-x_{n}\right\|}{\alpha_{n}}=\lim _{n \rightarrow \infty} \frac{\left\|x_{n+1}-x_{n}\right\|}{\rho_{n}} \cdot \frac{\rho_{n}}{\alpha_{n}}=0 .
$$


Hence, we conclude from (141) that for all $p \in \operatorname{VI}\left(\Omega, \widetilde{A}_{1}\right)$

$$
\begin{aligned}
& \left\langle\widetilde{A}_{2} p, w-p\right\rangle \\
& \quad=\lim _{j \rightarrow \infty}\left\langle\widetilde{A}_{2} p, x_{n_{j}}-p\right\rangle \\
& \quad \leq \limsup _{n \rightarrow \infty}\left\langle\widetilde{A}_{2} p, x_{n}-p\right\rangle \\
& \quad=\limsup _{n \rightarrow \infty}\left(\left\langle\widetilde{A}_{2} p, x_{n}-x_{n+1}\right\rangle+\left\langle\widetilde{A}_{2} p, x_{n+1}-p\right\rangle\right) \\
& \quad=\limsup _{n \rightarrow \infty}\left\langle\widetilde{A}_{2} p, x_{n+1}-p\right\rangle \\
& \leq 0 .
\end{aligned}
$$

That is,

$$
\left\langle\widetilde{A}_{2} p, p-w\right\rangle \geq 0, \quad \forall p \in \mathrm{VI}\left(\Omega, \widetilde{A}_{1}\right)
$$

Since $\widetilde{A}_{2}$ is $\beta$-strongly monotone and $L$-Lipschitz continuous, by Minty's Lemma [16], we know that (144) is equivalent to the VIP

$$
\left\langle\widetilde{A}_{2} w, p-w\right\rangle \geq 0, \quad \forall p \in \mathrm{VI}\left(\Omega, \widetilde{A}_{1}\right) .
$$

This shows that $w \in \operatorname{VI}\left(\operatorname{VI}\left(\Omega, \widetilde{A}_{1}\right), \widetilde{A}_{2}\right)$. Taking into account $\left\{x^{*}\right\}=\operatorname{VI}\left(\operatorname{VI}\left(\Omega, \widetilde{A}_{1}\right), \widetilde{A}_{2}\right)$, we know that $w=x^{*}$. Thus, $\omega_{w}\left(x_{n}\right)=\left\{x^{*}\right\}$; that is, $x_{n} \rightarrow x^{*}$.

Next we prove that $\lim _{n \rightarrow \infty}\left\|x_{n}-x^{*}\right\|=0$. As a matter of fact, utilizing (139) and (140) with $p=x^{*}$, we get

$$
\begin{aligned}
\| x_{n-1}- & x^{*} \|^{2} \\
\leq & \left(1-\alpha_{n} \tau\right)\left(\left\|v_{n}-x^{*}\right\|^{2}-2 \rho_{n}\left\langle\widetilde{A}_{1} v_{n}, \widetilde{v}_{n}-x^{*}\right\rangle\right) \\
& -2 \mu \alpha_{n}\left\langle\widetilde{A}_{2} x^{*}, x_{n+1}-x^{*}\right\rangle \\
\leq & \left(1-\alpha_{n} \tau\right)\left\|x_{n}-x^{*}\right\|^{2}-2 \rho_{n}\left\|\widetilde{A}_{1} v_{n}\right\|\left\|\widetilde{v}_{n}-x^{*}\right\| \\
& +2 \mu \alpha_{n}\left\langle\widetilde{A}_{2} x^{*}, x^{*}-x_{n+1}\right\rangle \\
= & \left(1-\alpha_{n} \tau\right)\left\|x_{n}-x^{*}\right\|^{2} \\
& +\alpha_{n} \tau \cdot \frac{2}{\tau}\left\{\frac{\rho_{n}}{\alpha_{n}}\left\|\widetilde{A}_{1} v_{n}\right\|\left\|\widetilde{v}_{n}-x^{*}\right\|\right. \\
& \left.+\mu\left\langle\widetilde{A}_{2} x^{*}, x^{*}-x_{n+1}\right\rangle\right\} .
\end{aligned}
$$

Since $\sum_{n=1}^{\infty} \alpha_{n}=\infty, \lim _{n \rightarrow \infty}\left(\rho_{n} / \alpha_{n}\right)=0$, and $\lim _{n \rightarrow \infty}\left\langle\widetilde{A}_{2} u^{*}, u^{*}-x_{n+1}\right\rangle=0$ (due to $x_{n} \rightarrow x^{*}$ ), we deduce that $\sum_{n=1}^{\infty} \alpha_{n} \tau=\infty$ and

$$
\lim _{n \rightarrow \infty} \frac{2}{\tau}\left\{\frac{\rho_{n}}{\alpha_{n}}\left\|\widetilde{A}_{1} v_{n}\right\|\left\|\widetilde{v}_{n}-u^{*}\right\|+\mu\left\langle\widetilde{A}_{2} u^{*}, u^{*}-x_{n+1}\right\rangle\right\}=0 .
$$

Therefore, applying Lemma 10 to (146), we infer that $\lim _{n \rightarrow \infty}\left\|x_{n}-x^{*}\right\|=0$. This completes the proof.
Remark 20. It is obvious that iterative scheme (48) is very different from Yao et al. iterative one (14) and Zeng et al. iterative one in Algorithm ZWY. Here, the two-step iterative scheme in [11, Algorithm 3.2] is extended to develop fourstep iterative scheme (48) for the THVI (19) by combining Korpelevich's extragradient method, hybrid steepest-descent method, and Mann's iteration method. It is worth pointing out that under the lack of the assumptions similar to those in $\left[8\right.$, Theorem 3.2], for example, $\left\{x_{n}\right\}$ is bounded, $\operatorname{Fix}(T) \cap$ int $C \neq \emptyset$, and $\|x-T x\| \geq k \operatorname{Dist}(x, \operatorname{Fix}(T)), \forall x \in C$, for some $k>0$, the sequence $\left\{x_{n}\right\}$ generated by (48) converges strongly to a point $u^{*} \in \bigcap_{n=1}^{\infty} \operatorname{Fix}\left(T_{n}\right) \cap \bigcap_{k=1}^{M} \operatorname{GMEP}\left(\Theta_{k}, \varphi_{k}, A_{k}\right) \cap$ $\bigcap_{i=1}^{N} I\left(B_{i}, R_{i}\right) \cap \operatorname{GSVI}(G)=: \Omega$, which is a unique solution of the VIP: $\left\langle\widetilde{A}_{2} u^{*}, p-u^{*}\right\rangle \geq 0, \forall p \in \Omega$.

Remark 21. Theorems 18 and 19 improve, extend, supplement, and develop Yao et al. [8, Theorems 3.1 and 3.2] and Zeng et al. [11, Theorem 3.2] in the following aspects.

(a) THVI (19) with the unique solution $x^{*} \in \Omega$ satisfying

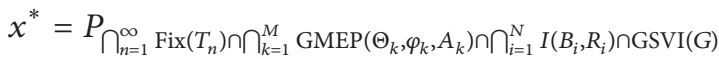

$$
\begin{aligned}
& \cdot\left(I-\widetilde{A_{1}}\right) x^{*}
\end{aligned}
$$

is more general than the problem of finding a point $\tilde{x} \in C$ satisfying $\tilde{x}=P_{\operatorname{Fix}(T)} S \tilde{x}$ in [8] and than the problem of finding a point $x^{*} \in \bigcap_{i=1}^{N} \operatorname{Fix}\left(T_{i}\right)$ satisfying $x^{*}=P_{\bigcap_{i=1}^{N} \operatorname{Fix}\left(T_{i}\right)}(I-$ $\left.A_{1}\right) x^{*}$ in [11, Theorem 3.2]. It is worth pointing out that $S$ is nonexpansive if and only if the complement $I-S$ is $(1 / 2)$ inverse strongly monotone; see [25].

(b) Four-step iterative scheme (48) for THVI (19) is more flexible, more advantageous, and more subtle than Zeng et al. two-step iterative one in [11, Algorithm 3.2] and than Yao et al. two-step iterative one (14) because it can be used to solve several kinds of problems, for example, the THVI, the hierarchical VIP, and the problem of finding a common point of four sets: $\bigcap_{n=1}^{\infty} \operatorname{Fix}\left(T_{n}\right), \bigcap_{k=1}^{M} \operatorname{GMEP}\left(\Theta_{k}, \varphi_{k}, A_{k}\right)$, $\bigcap_{i=1}^{N} I\left(B_{i}, R_{i}\right)$, and $\operatorname{GSVI}(G)$. In addition, it also drops the crucial requirements that $\operatorname{Fix}(T) \cap \operatorname{int} C \neq \emptyset$ and $\|x-T x\| \geq$ $k \operatorname{Dist}(x, \operatorname{Fix}(T)), \forall x \in C$, for some $k>0$ in [8, Theorem 3.2 (v)].

(c) The argument techniques in Theorems 18 and 19 are very different from the argument ones in [8, Theorems 3.1 and 3.2] and from the argument ones in [11, Theorem 3.2] because we use the $W$-mapping approach to fixed points of infinitely many nonexpansive mappings $\left\{T_{n}\right\}_{n=1}^{\infty}$ (see Lemmas 7 and 8), the properties of resolvent operators and maximal monotone mappings, the fixed point equation $x^{*}=P_{C}\left(I-v_{1} F_{1}\right) P_{C}(I-$ $\left.v_{2} F_{2}\right) x^{*}$ equivalent to the GSVI (10) (see Proposition CWY), and the contractive coefficient estimates for the contractions associated with nonexpansive mappings (see Lemma 9).

(d) Compared with the restrictions on the parameter sequences of [8, Theorem 19] and [11, Theorem 3.2], respectively, the hypotheses (H3)-(H5) in Theorem 18 are additionally added because Theorem 18 involves the quite complex problem, that is, the THVI (19) (over the set $\bigcap_{n=1}^{\infty} \operatorname{Fix}\left(T_{n}\right)$ ) 
with constraints of several problems: finitely many GMEPs, finitely many variational inclusions, and GSVI (10).

\section{Conflict of Interests}

The authors declare that there is no conflict of interests regarding the publication of this paper.

\section{Acknowledgment}

This paper was funded by the Deanship of Scientific Research (DSR), King Abdulaziz University, Jeddah. The authors, therefore, acknowledge with thanks DSR for the technical and financial support.

\section{References}

[1] J.-L. Lions, Quelques Méthodes de Résolution des Problèmes aux Limites non Linéaires, Dunod, Paris, Farnce, 1969.

[2] G. M. Korpelevich, "The extragradient method for finding saddle points and other problems," Matecon, vol. 12, pp. 747756, 1976.

[3] J. W. Peng and J. C. Yao, "A new hybrid-extragradient method for generalized mixed equilibrium problems, fixed point problems and variational inequality problems," Taiwanese Journal of Mathematics, vol. 12, no. 6, pp. 1401-1432, 2008.

[4] L. C. Ceng, S. M. Guu, and J. C. Yao, "Hybrid viscosity CQ method for finding a common solution of a variational inequality, a general system of variational inequalities, and a fixed point problem," Fixed Point Theory and Applications, vol. 2013, article 313, 2013.

[5] Y. Yao, Y. C. Liou, and S. M. Kang, "Approach to common elements of variational inequality problems and fixed point problems via a relaxed extragradient method," Computers \& Mathematics with Applications, vol. 59, no. 11, pp. 3472-3480, 2010.

[6] L.-C. Ceng, C.-Y. Wang, and J.-C. Yao, "Strong convergence theorems by a relaxed extragradient method for a general system of variational inequalities," Mathematical Methods of Operations Research, vol. 67, no. 3, pp. 375-390, 2008.

[7] R. U. Verma, "On a new system of nonlinear variational inequalities and associated iterative algorithms," Mathematical Sciences Research Hot-Line, vol. 3, no. 8, pp. 65-68, 1999.

[8] Y. Yao, Y.-C. Liou, and G. Marino, “Two-step iterative algorithms for hierarchical fixed point problems and variational inequality problems," Journal of Applied Mathematics and Computing, vol. 31, no. 1-2, pp. 433-445, 2009.

[9] H. Iiduka, "Strong convergence for an iterative method for the triple-hierarchical constrained optimization problem," Nonlinear Analysis: Theory, Methods \& Applications, vol. 71, no. 12, pp. e1292-e1297, 2009.

[10] H. Iiduka, "Iterative algorithm for solving triple-hierarchical constrained optimization problem," Journal of Optimization Theory and Applications, vol. 148, no. 3, pp. 580-592, 2011.

[11] L. C. Zeng, M. M. Wong, and J. C. Yao, "Strong convergence of relaxed hybrid steepest-descent methods for triple hierarchical constrained optimization," Fixed Point Theory and Applications, vol. 2012, article 29, 24 pages, 2012.

[12] A. Latif, A. E. Al-Mazrooei, B. A. B. Dehaish, and J. C. Yao, "Hybrid viscosity approximation methods for general systems of variational inequalities in Banach spaces," Fixed Point Theory and Applications, vol. 2013, article 258, 2013.

[13] L. C. Ceng, A. Latif, Q. H. Ansari, and J.-C. Yao, "Hybrid extragradient method for hierarchical variational inequalities," Fixed Point Theory and Applications, vol. 2014, article 222, 2014.

[14] Q. H. Ansari, L.-C. Ceng, and H. Gupta, Triple Hierarchical Variational Inequalities in Nonlinear Analysis: Approximation Theory, Optimization and Applications, Springer, New York, NY, USA, 2014.

[15] L.-C. Ceng and J.-C. Yao, "A hybrid iterative scheme for mixed equilibrium problems and fixed point problems," Journal of Computational and Applied Mathematics, vol. 214, no. 1, pp. 186201, 2008.

[16] K. Goebel and W. A. Kirk, Topics on Metric Fixed-Point Theory, Cambridge University Press, Cambridge, UK, 1990.

[17] J. G. O’Hara, P. Pillay, and H. K. Xu, "Iterative approaches to convex feasibility problems in Banach spaces," Nonlinear Analysis: Theory, Methods \& Applications, vol. 64, no. 9, pp. 2022-2042, 2006.

[18] H. K. Xu and T. H. Kim, "Convergence of hybrid steepestdescent methods for variational inequalities," Journal of Optimization Theory and Applications, vol. 119, no. 1, pp. 185-201, 2003.

[19] R. T. Rockafellar, "Monotone operators and the proximal point algorithm," SIAM Journal on Control and Optimization, vol. 14, no. 5, pp. 877-898, 1976.

[20] V. Barbu, Nonlinear Semigroups and Differential Equations in Banach Spaces, Noordhoff, Groningen, The Netherlands, 1976.

[21] N.-J. Huang, "A new completely general class of variational inclusions with noncompact valued mappings," Computers \& Mathematics with Applications, vol. 35, no. 10, pp. 9-14, 1998.

[22] L.-C. Ceng, Q. H. Ansari, M. M. Wong, and J.-C. Yao, "Mann type hybrid extragradient method for variational inequalities, variational inclusions and fixed point problems," Fixed Point Theory, vol. 13, no. 2, pp. 403-422, 2012.

[23] L.-C. Zeng, S.-M. Guu, and J.-C. Yao, "Characterization of $H$-monotone operators with applications to variational inclusions," Computers \& Mathematics with Applications, vol. 50, no. 3-4, pp. 329-337, 2005.

[24] Y. Yao, Y.-C. Liou, and J.-C. Yao, "Convergence theorem for equilibrium problems and fixed point problems of infinite family of nonexpansive mappings," Fixed Point Theory and Applications, vol. 2007, Article ID 64363, 12 pages, 2007.

[25] C. Byrne, "A unified treatment of some iterative algorithms in signal processing and image reconstruction," Inverse Problems, vol. 20, no. 1, pp. 103-120, 2004. 


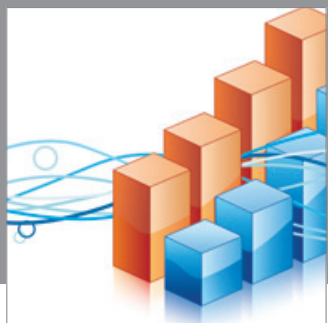

Advances in

Operations Research

mansans

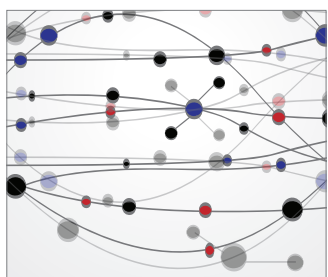

The Scientific World Journal
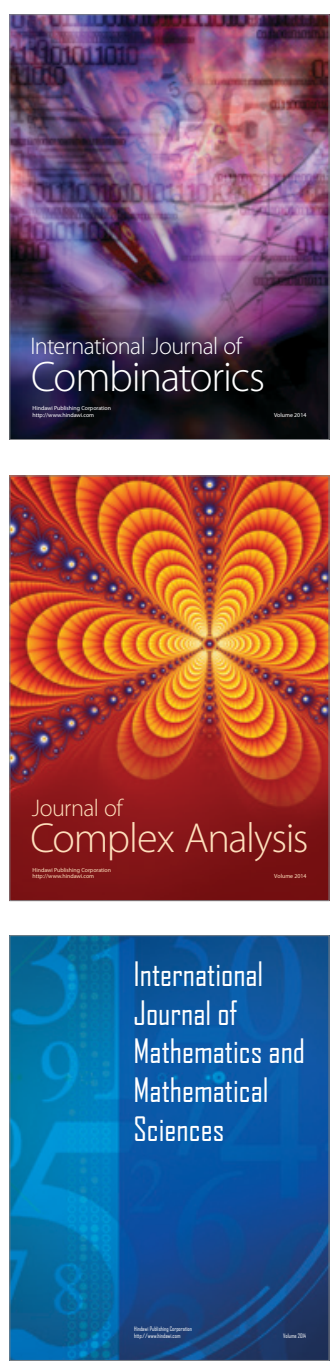
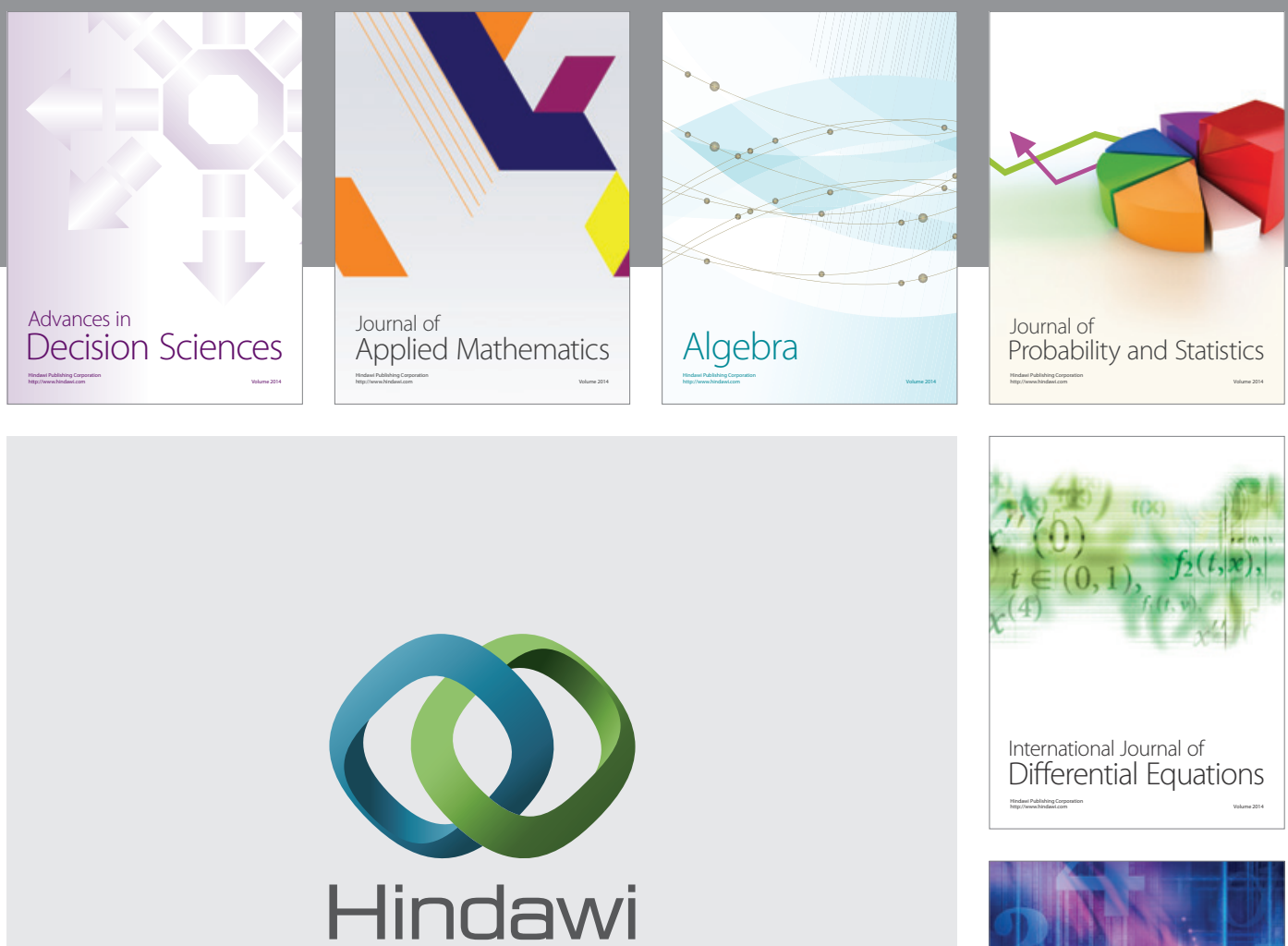

Submit your manuscripts at http://www.hindawi.com
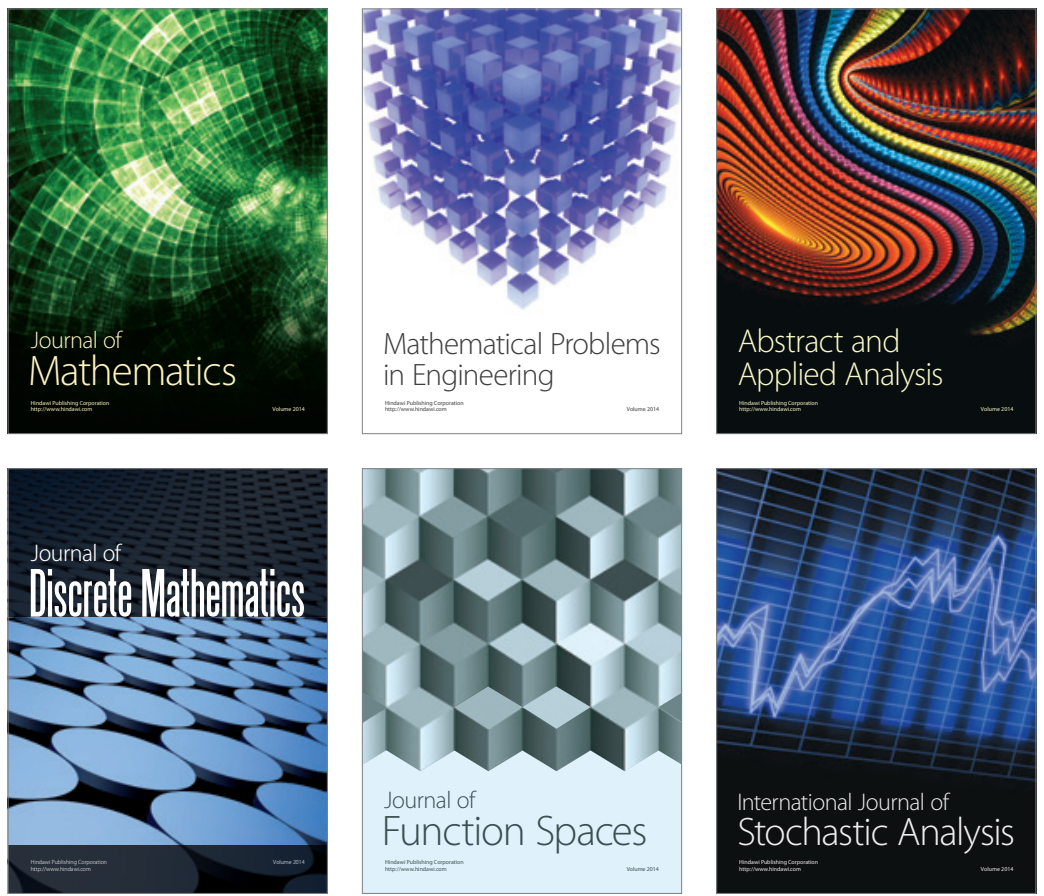

Journal of

Function Spaces

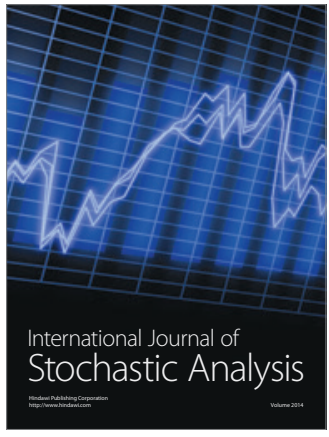

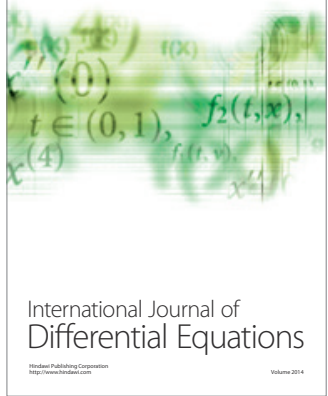
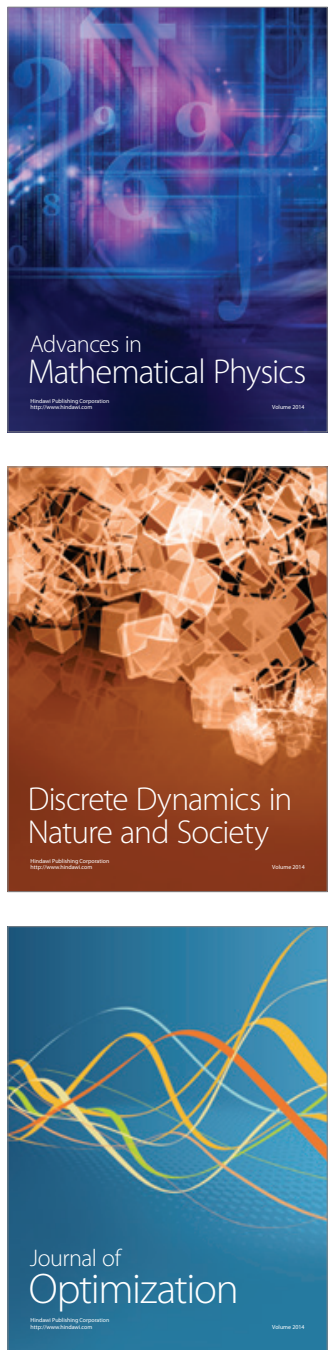